\title{
Characterization of Introgression Lines for Yield-related Traits with Indica-type Rice Variety IR64 Genetic Background
}

\author{
Daisuke FUJITA ${ }^{1}$, Rizza Eve M. SANTOS ${ }^{1}$, Leodegario A. EBRON ${ }^{1}$, \\ Mary J. TELEBANCO- YANORIA ${ }^{1}$, Hiroshi KATO ${ }^{2}$, Sohei KOBAYASHI ${ }^{3}$, \\ Yusaku UGA ${ }^{4}$, Etsuko ARAKI ${ }^{5}$, Toshiyuki TAKAI ${ }^{2}$, Hiroshi TSUNEMATSU ${ }^{2}$, \\ Tokio IMBE ${ }^{6}$, Gurdev S. KHUSH ${ }^{1}$, Darshan S. BRAR ${ }^{1}$, Yoshimichi FUKUTA ${ }^{7}$ \\ and Nobuya KOBAYASHI ${ }^{1,7^{*}}$ \\ 1 International Rice Research Institute (DAPO Box 7777, Metro Manila, Philippines) \\ 2 National Institute of Crop Science, National Agriculture and Food Research Organization \\ (NARO) (Tsukuba, Ibaraki 305-8518, Japan) \\ 3 National Agricultural Research Center for Hokkaido Region, NARO (Sapporo, Hokkaido \\ 062-8555, Japan) \\ 4 National Institute of Agrobiological Sciences (Tsukuba, Ibaraki 305-8602, Japan) \\ 5 National Agricultural Research Center for Western Region, NARO (Fukuyama, Hiroshima \\ 721-8514, Japan) \\ 6 National Agricultural Research Center for Kyushu Okinawa Region, NARO (Koshi, Kumamoto \\ 861-1192, Japan) \\ 7 Japan International Research Center for Agricultural Sciences (Tsukuba, Ibaraki 305-8686, \\ Japan)
}

\begin{abstract}
A total of 334 introgression lines (INLs: $\mathrm{BC}_{3}$-derived lines) derived from crosses between Indica cultivar IR64 as a recurrent parent and 10 donor parents including new plant type lines (IR6560087-2-2-3, IR65598-112-2, IR65564-2-2-3, IR66750-6-2-1, IR69093-41-2-3-2, IR69125-25-3-1-1, Hoshiaoba, IR66215-44-2-3, IR68522-10-2-2, and IR71195-AC1) have been developed by recurrent backcross breeding, to introduce unique traits into IR64 genetic background and to use for breeding materials. The agronomic traits of the 334 INLs were evaluated in International Rice Research Institute from 2005 to 2007 and the genotypes of the 334 INLs were detected using SSR markers. Particularly, 117 out of the 334 INLs which showed unique agronomic traits were selected as the "core set" for detailed evaluation. Variation in agronomic traits: days to heading (DTH), leaf length and width, culm and panicle length, number of panicles, total spikelet number per panicle (TSN), and 100-grain weight (GW), in the 117 INLs were characterized. As a result of comparison between the several INLs and IR64, significant differences were observed in DTH, TSN and GW in at least one season. The developed INLs with the IR64 genetic background are useful for both breeding and genetic dissection of agronomic traits across various environments.
\end{abstract}

Discipline: Plant breeding

Additional key words: days to heading, grain weight, new plant type, Oryza sativa L., total spikelet number

\section{Introduction}

Since the 1960s, many International Rice Research Institute (IRRI)-bred varieties have been released and distributed worldwide and used by farmers as cultivated varieties and breeders as important parental varieties in breeding programs. IRRI has developed many highyielding Indica inbred cultivars such as IR8, IR36, IR 64 , and IR72 ${ }^{20}$. Especially, IR64, which was released in 1985, had been widely accepted as a high-quality rice variety in many countries ${ }^{7}$. To increase yield potential in inbred varieties under a tropical environment, new plant

\section{Background:}

This paper reports the results obtained in the IRRI-Japan Collaborative Research Project phases III, IV and V supported by the Ministry of Foreign Affairs and the Ministry of Agriculture, Forestry and Fisheries of Japan.

*Corresponding author: e-mail nobuya@affrc.go.jp

Received 3 June 2009; accepted 19 October 2009. 
type (NPT) rice have been developed using tropical Japonica varieties. The NPT lines have unique agronomic traits from tropical Japonica such as low tillering, large panicles, thick culm, and large and dark green flag leaves ${ }^{19}$

Because of wide acceptability of IR64, several breeding materials with IR64 genetic background such as doubled haploid (DH) lines ${ }^{3}$, recombinant inbred lines (RILs), and thousands of mutant lines ${ }^{24}$ have been developed for genetic analysis and improvement of rice varieties. Additionally, recent progress in molecular biology has made it possible to conduct genetic analysis in complex agronomic traits which were governed by multiple quantitative trait loci (QTLs) and their interactions with other loci. For example, $135 \mathrm{DH}$ lines derived via anther culture from a cross between IR64 and tropical Japonica cultivar Azucena were developed and the DH lines were used for QTL analysis of grain shape ${ }^{6}$, panicle number ${ }^{12}$, blast resistance ${ }^{22}$, and brown planthopper resistance $^{6}$. These materials with IR64 genetic background are useful as not only the materials for detecting QTLs in genetic analysis but also breeding materials which are similar in elite traits to IR64.

Introgression lines (INLs) derived from crosses between IR64 and 10 donor varieties which were mostly NPT lines have been developed in the present study as breeding materials for enhancement of rice yield ${ }^{2}$. A total of 334 INLs were selected by agronomic traits related to yield such as days to heading, total spikelet number and grain size. Based on characterization of phenotype and genotype in the 334 INLs, associations between agronomic traits and introgressed chromosomal segments were investigated. A total of 54 regions for eight traits were associated with agronomic traits: seven regions for days to heading (DTH), eight regions for culm length (CL), eight regions for leaf width (LW), four regions for leaf length (LL), six regions for panicle length (PL), three regions for panicle number $(\mathrm{PN})$, seven regions for 100-grain weight $(\mathrm{GW})$, and 11 regions for total spikelet number per panicle (TSN). These lines with IR64 genetic background are useful as research and breeding materials to enhance yield potential of IR64 using marker-assisted selection (MAS) in breeding programs.

In this study, 117 lines representing diversity of 334 INLs derived from crosses between IR64 and the 10 donors were characterized for agronomic traits: DTH, CL, PL, LW, LL, PN, TSN, and GW. Especially, several INLs which have unique agronomic traits such as DTH, TSN and GW, were compared with IR64.

\section{Materials and methods}

\section{Plant materials}

A total of 334 INLs were developed by the backcross breeding in the combinations between an Indicatype variety IR64 as a recurrent parent and 10 donor varieties: IR65600-87-2-2-3 (YP1), IR65598-112-2 (YP3), IR65564-2-2-3 (YP4), IR69093-41-2-3-2 (YP5), IR 69125-25-3-1-1 (YP6), Hoshiaoba (YP7), IR66215-442-3 (YP8), IR68522-10-2-2 (YP9), IR71195-AC1 (YP 10), and IR66750-6-2-1 (YP11) (Table 1). Among the

Table 1. List of $\mathbf{1 0}$ donor varieties which were used for development of introgression lines.

\begin{tabular}{lllcc}
\hline \hline Entry No. & Donor variety & Parents' varieties for donor & \multicolumn{2}{c}{ Number of INLs } \\
\cline { 3 - 5 } & & Developed & Core set \\
\hline YP1 & IR65600-87-2-2-3 & Shen Nung 89-366, Ketan Lumbu & 36 & 10 \\
YP3 & IR65598-112-2 & Shen Nung 89-366, Genjah Wangkal & 23 & 12 \\
YP4 & IR65564-2-2-3 & NO 11, Bali Ontjer & 45 & 19 \\
YP5 & IR69093-41-2-3-2 & Shen Nung 89-366, Ketan Lumbu, Gundil Kuning & 56 & 19 \\
YP6 & IR69125-25-3-1-1 & Shen Nung 89-366, Ketan Lumbu, Gundil Kuning & 29 & 7 \\
YP7 & Hoshiaoba & Chugoku 113, Oochikara & 21 & 11 \\
YP8 & IR66215-44-2-3 & Gaok, Chir 87-3-1, Moroberekan, Palawan & 29 & 10 \\
YP9 & IR68522-10-2-2 & Moroberekan, Shen Nung 89-366, Daringan & 16 & 7 \\
YP10 & IR71195-AC1 & Shen Nung 89-366, Pring, Akihikari, Cnax 1419-37-2-3-4, Mee Nteri & 39 & 11 \\
YP11 & IR66750-6-2-1 & Shen Nung 89-366, Sri Kuning & 40 & 11 \\
\hline
\end{tabular}

The underline indicates variety from Indonesia (tropical Japonica).

Donors were developed from the crossing among several varieties including Japonica-type. 
10 donor varieties, nine which were designated with the IR number were the NPT varieties developed in IRRI using tropical Japonica-type varieties ${ }^{19}$. The other one donor parent, Hoshiaoba ${ }^{14}$ (also known as Chugoku 146), was a high-yielding variety developed in Japan and derived from a cross between Indica and Japonicatypes' varieties. These lines and a variety were used as donor parents to enhance yield potential.

The $F_{1}$ plants which were derived from the crosses between IR64 and these donor varieties were recurrently backcrossed with IR64 for three times. Several $\mathrm{BC}_{3} \mathrm{~F}_{1}$ individuals were selected by agronomic traits related to yield such as heading date, tiller number, plant height, panicle size, and grain size. The selection of $\mathrm{BC}_{3} \mathrm{~F}_{1}$ individuals was conducted for selecting the individuals with unique agronomic traits related to yield. A single $\mathrm{BC}_{3} \mathrm{~F}_{1}$ plant from each donor parent was continuously selfpollinated and selected for maintaining variation of these traits in every generation by the $\mathrm{BC}_{3} \mathrm{~F}_{7}$ in 2005 . The backcrossed inbred lines derived from crosses between IR64 and 10 donor parents were developed although the number of backcrossed inbred lines from each donor was small. The group of backcrossed inbred lines from each donor was called a "sib group". A total of 334 INLs were developed and consisted of 36 lines from YP1 (YTH 2-38), 23 lines from YP3 (YTH 5578), 45 lines from YP4 (YTH 79-126), 56 lines from YP 5 (YTH 169-227), 29 lines from YP6 (YTH 229-258), 21 lines from YP7 (YTH 259-280), 29 lines from YP8 (YTH 282-311), 16 lines from YP9 (YTH 312-328), 39 lines from YP10 (YTH 329-369), and 40 lines from YP 11 (YTH 127-168) (Table 2).

\section{Phenotypic evaluation for agronomic traits}

The 334 INLs were grown at the field of IRRI, Los Baños, Laguna, Philippines, in the dry seasons (DS: January to May) and the wet seasons (WS: July to November) from 2005 to 2007. A total of 117 lines which represented variation of agronomic traits in the 334 INLs were also grown at the field of IRRI in the DS and the WS in 2008. Each line was represented by more than two rows of 12 individuals. A single plant at 21days after sowing was transplanted at $20 \mathrm{~cm}$ between hills and at $30 \mathrm{~cm}$ between rows. A total of eight agronomic traits in the 117 INLs were evaluated from 2005 to 2008. The six traits: DTH, CL, PL, LW, LL, and PN, were evaluated in 2005WS, 2006DS, 2006WS, 2007DS, 2007WS, and 2008DS. Two yield components: TSN and GW were evaluated in 2007DS, 2007WS and 2008DS.

DTH was evaluated as the number of days from sowing seeds until $50 \%$ of the panicles flowered. A single value per plant of 10 individuals which were planted in the middle of each line were measured for CL, PL, LW, LL, PN, TSN, and GW. CL was measured from the soil surface to the neck of the tallest tiller in a plant. PL was measured from panicle neck to the panicle tip of the tallest tiller. LW and LL were measured on the second leaf under the flag leaf of the tallest tiller. PN was counted as the number of productive panicles per plant. TSN was calculated as the sum of filled and unfilled spikelets per panicle. GW was measured as the weight of 100 filled grains for each plant. The averages of agronomic traits in the 117 INLs were calculated in 2005, 2006, 2007, and 2008. The averages of agronomic traits for DS were calculated using the data of DS in 2006, 2007 and 2008. Similarly, the averages of agronomic traits for WS were calculated based on the data of WS in 2005, 2006 and 2007. The averages of TSN and GW for DS in 2008 were used as the representative data of DS and those for WS in 2007 were used as the representative data of WS.

\section{Genotyping using SSR markers}

The whole genome DNA of the $334 \mathrm{BC}_{3} \mathrm{~F}_{8}$ plants was individually extracted from fresh leaves using the CTAB method ${ }^{21}$. DNA of these INLs was analyzed using simple sequence repeat (SSR) markers ${ }^{15}$ distributed across the 12 rice chromosomes. A total of 457 SSR markers of known chromosomal position were used to survey polymorphism between IR64 and 10 donor parents. More than 200 polymorphic markers for each sib group: 247 markers for YP1-INLs, 248 for YP3-INLs, 261 for YP4-INLs, 262 for YP5-INLs, 260 for YP6INLs, 238 for YP7-INLs, 224 for YP8-INLs, 266 for YP 9-INLs, 280 for YP10-INLs, and 276 for YP11-INLs, were used for genotyping of the INLs.

To detect the introgressed segments of donor parents, genotypes of the 334 INLs were analyzed using the polymorphic SSR markers. Firstly, the genotypes of postulated $\mathrm{BC}_{3} \mathrm{~F}_{1}$ plants were analyzed using bulked DNA of sib INLs for each donor parent. The genotypes of 10 bulked DNA from each sib group were detected using about 200 polymorphic SSR markers. Secondly, the genotypes of the 334 INLs have been individually analyzed using the SSR markers which detected introgressed segment regions in the bulked DNA.

PCR amplification was conducted using a DNA engine dyad thermal cycler (Bio-Rad). The $15 \mu$ PCR reaction mixture contained $50 \mathrm{mM} \mathrm{KCl}, 10 \mathrm{mM}$ Tris- $\mathrm{HCl}$ (pH 9.0), $1.5 \mathrm{mM} \mathrm{MgCl}, 200 \mu \mathrm{M}$ dNTP, $0.2 \mu \mathrm{M}$ primer, 1 unit of Taq polymerase (SBS Genetech, China), and $5-10 \mu \mathrm{g} / \mathrm{ml}$ of genomic DNA as a template. The thermal cycler was programmed for a first denaturation step of $5 \mathrm{~min}$ at $95^{\circ} \mathrm{C}$, followed by 35 cycles, 
D. Fujita et al.

Table 2. List of 334 introgression lines (INLs) bred from the crosses between IR64 as a recurrent variety and 10 different donor varieties.

\begin{tabular}{|c|c|c|c|c|c|c|c|}
\hline $\begin{array}{l}\text { Entry No. } \\
\text { in 2005WS }\end{array}$ & IR designation number & $\begin{array}{c}\text { Donor } \\
\text { parenta) }\end{array}$ & $\begin{array}{c}\text { Core } \\
\text { set }\end{array}$ & $\begin{array}{l}\text { Entry No. } \\
\text { in } 2005 \mathrm{WS}\end{array}$ & IR designation number & $\begin{array}{l}\text { Donor } \\
\text { parent }\end{array}$ & $\begin{array}{c}\text { Core } \\
\text { set }\end{array}$ \\
\hline YTH 2 & IR84633-9-5-2-2-1-4-2-2-B & YP1 & & YTH 79 & IR84642-8-1-2-2-3-2-2-2-B & YP4 & \\
\hline YTH 3 & IR84633-9-5-2-4-4-3-2-2-B & YP1 & & YTH 80 & IR84642-8-1-2-6-1-2-4-3-2-2-B & YP4 & \\
\hline YTH 4 & IR84633-9-9-7-2-1-3-2-3-B & YP1 & & YTH 82 & IR84642-8-1-2-7-2-3-2-2-B & YP4 & \\
\hline YTH 5 & IR84633-9-9-7-6-1-4-2-2-B & YP1 & & YTH 83 & IR84642-8-4-3-4-4-2-4-2-2-6-B & YP4 & $\mathrm{X}$ \\
\hline YTH 6 & IR84633-9-9-8-3-3-3-2-2-B & YP1 & $\mathrm{X}$ & YTH 84 & IR84642-8-4-3-11-2-2-5-2-2-2-B & YP4 & $\mathrm{X}$ \\
\hline YTH 7 & IR84633-9-9-8-8-3-3-2-2-B & YP1 & & YTH 85 & IR84642-8-4-3-5-2-2-4-2-2-2-B & YP4 & $\mathrm{X}$ \\
\hline YTH 8 & IR84633-9-12-3-4-1-2-2-2-B & YP1 & & YTH 86 & IR84642-8-4-3-10-5-2-4-5-2-2-B & YP4 & \\
\hline YTH 9 & IR84633-9-12-3-8-1-2-2-3-B & YP1 & & YTH 87 & IR84642-8-6-4-6-3-4-2-2-B & YP4 & \\
\hline YTH 10 & IR84633-9-14-4-5-3-3-2-2-B & YP1 & $\mathrm{X}$ & YTH 88 & IR84642-8-6-6-3-4-3-4-2-2-2-B & YP4 & $\mathrm{X}$ \\
\hline YTH 11 & IR84633-9-14-4-10-3-3-2-2-B & YP1 & & YTH 89 & IR84642-8-6-6-8-3-3-2-3-B & YP4 & $\mathrm{X}$ \\
\hline YTH 12 & IR84633-9-14-6-4-1-3-2-2-B & YP1 & $\mathrm{X}$ & YTH 90 & IR84642-8-23-4-7-3-3-2-2-B & YP4 & \\
\hline YTH 13 & IR84633-9-14-6-8-4-3-2-2-B & YP1 & $\mathrm{X}$ & ҮТН 91 & IR84642-8-23-4-8-2-2-4-2-2-2-B & YP4 & \\
\hline YTH 14 & IR84633-9-16-5-7-3-2-4-2-2-2-B & YP1 & & YTH 92 & IR84642-8-35-10-6-2-2-4-2-2-2-B & YP4 & $\mathrm{X}$ \\
\hline YTH 15 & IR84633-9-16-5-11-2-2-4-3-2-2-B & YP1 & $\mathrm{X}$ & YTH 93 & IR84642-8-36-8-7-2-2-5-2-2-2-B & YP4 & \\
\hline YTH 16 & IR84633-9-28-3-9-2-5-2-3-B & YP1 & $\mathrm{X}$ & YTH 94 & IR84642-8-36-8-8-2-2-4-2-2-3-B & YP4 & \\
\hline YTH 17 & IR84633-9-28-10-8-2-3-5-2-2-2-B & YP1 & & YTH 95 & IR84642-8-36-10-2-2-2-4-2-2-3-B & YP4 & \\
\hline YTH 18 & IR84633-9-50-2-3-2-3-2-2-B & YP1 & & YTH 96 & IR84642-8-36-10-6-3-2-5-2-2-2-B & YP4 & \\
\hline YTH 19 & IR84633-9-50-2-6-2-4-2-2-B & YP1 & & YTH 97 & IR84642-8-49-6-11-4-3-2-3-B & YP4 & \\
\hline YTH 20 & IR84633-9-50-3-6-2-2-2-5-B & YP1 & $\mathrm{X}$ & YTH 98 & IR84642-8-61-8-9-2-3-2-4-B & YP4 & \\
\hline YTH 22 & IR84633-9-50-3-10-1-2-2-9-B & YP1 & & YTH 99 & IR84642-8-61-8-10-4-2-4-2-2-2-B & YP4 & $\mathrm{X}$ \\
\hline YTH 23 & IR84633-9-58-6-3-3-2-4-2-2-2-B & YP1 & & YTH 100 & IR84642-8-61-11-4-3-2-4-2-2-4-B & YP4 & \\
\hline YTH 24 & IR84633-9-58-6-6-2-2-5-2-2-2-B & YP1 & $X$ & YTH 102 & IR84642-8-61-11-8-3-2-4-2-2-2-B & YP4 & \\
\hline YTH 25 & IR84633-9-58-6-8-1-2-4-2-2-3-B & YP1 & & YTH 103 & IR84642-8-68-2-3-2-2-4-2-2-2-B & YP4 & \\
\hline YTH 26 & IR84633-9-64-7-5-1-3-2-2-B & YP1 & & YTH 104 & IR84642-8-68-2-6-2-2-4-2-2-2-B & YP4 & \\
\hline YTH 27 & IR84633-9-77-3-10-3-3-2-2-B & YP1 & & YTH 105 & IR84642-8-68-2-10-3-2-4-2-2-2-B & YP4 & $\mathrm{X}$ \\
\hline YTH 28 & IR84633-9-77-10-5-2-2-4-2-3-2-B & YP1 & & YTH 106 & IR84642-8-68-11-5-2-2-4-3-2-2-B & YP4 & \\
\hline YTH 29 & IR84633-9-77-10-7-1-2-4-2-2-3-B & YP1 & & YTH 107 & IR84642-8-68-11-9-2-4-2-4-B & YP4 & $\mathrm{X}$ \\
\hline YTH 30 & IR84633-9-92-4-4-3-3-2-5-B & YP1 & & YTH 108 & IR84642-8-97-3-3-3-2-4-2-2-2-B & YP4 & $\mathrm{X}$ \\
\hline YTH 31 & IR84633-9-92-4-10-3-3-2-2-B & YP1 & & YTH 109 & IR84642-8-97-3-9-2-2-4-2-2-2-B & YP4 & \\
\hline YTH 32 & IR84633-9-92-5-3-3-2-4-2-2-2-B & YP1 & & YTH 110 & IR84642-8-97-8-6-3-2-4-2-2-2-B & YP4 & \\
\hline YTH 33 & IR84633-9-92-5-2-3-2-4-2-2-2-B & YP1 & $\mathrm{X}$ & YTH 111 & IR84642-8-106-3-5-3-4-4-2-2-2-B & YP4 & \\
\hline YTH 34 & IR84633-9-92-10-4-2-3-2-2-B & YP1 & & YTH 112 & IR84642-8-106-3-9-2-3-2-4-B & YP4 & $\mathrm{X}$ \\
\hline YTH 35 & IR84633-9-94-2-2-2-3-2-2-B & YP1 & $\mathrm{X}$ & YTH 113 & IR84642-8-106-3-11-3-3-2-4-B & YP4 & $\mathrm{X}$ \\
\hline YTH 36 & IR84633-9-94-2-11-2-3-2-2-B & YP1 & & YTH 114 & IR84642-8-106-6-2-2-2-4-1-2-2-B & YP4 & $\mathrm{X}$ \\
\hline YTH 37 & IR84633-9-107-10-5-3-3-2-2-B & YP1 & & YTH 115 & IR84642-8-106-6-10-2-3-2-2-B & YP4 & \\
\hline YTH 38 & IR84633-9-107-10-8-1-2-5-2-2-2-B & YP1 & & YTH 116 & IR84642-8-106-6-11-2-3-2-2-B & YP4 & \\
\hline YTH 55 & IR84635-10-10-10-3-3-2-4-2-2-2-B & YP3 & & YTH 117 & IR84642-8-106-7-4-3-3-2-3-B & YP4 & \\
\hline YTH 56 & IR84635-10-10-10-9-5-3-2-2-B & YP3 & & YTH 118 & IR84642-8-106-7-6-2-2-4-3-2-2-B & YP4 & $\mathrm{X}$ \\
\hline YTH 57 & IR84635-10-18-7-10-4-2-2-2-B & YP3 & $\mathrm{X}$ & YTH 119 & IR84642-8-106-7-11-3-3-2-2-B & YP4 & $\mathrm{X}$ \\
\hline YTH 58 & IR84635-10-20-2-4-1-2-2-B & YP3 & $\mathrm{X}$ & YTH 120 & IR84642-8-115-2-3-3-4-2-2-B & YP4 & \\
\hline YTH 59 & IR84635-10-20-2-7-1-2-2-B & YP3 & $\mathrm{X}$ & YTH 122 & IR84642-8-115-2-6-2-3-2-2-B & YP4 & $\mathrm{X}$ \\
\hline YTH 60 & IR84635-10-44-7-1-3-4-2-2-2-B & YP3 & $\mathrm{X}$ & YTH 123 & IR84642-8-119-3-5-2-3-2-2-B & YP4 & $\mathrm{X}$ \\
\hline YTH 62 & IR84635-10-44-7-4-5-4-3-2-2-B & YP3 & & YTH 124 & IR84642-8-119-3-9-2-3-2-2-B & YP4 & $\mathrm{X}$ \\
\hline YTH 63 & IR84635-10-59-4-2-2-3-4-2-2-8-B & YP3 & $\mathrm{X}$ & YTH 125 & I884642-8-119-10-8-3-2-4-2-2-2-B & YP4 & $\mathrm{X}$ \\
\hline YTH 64 & IR84635-10-59-4-7-2-2-4-2-2-14-B & YP3 & & YTH 126 & IR84642-8-119-10-10-3-2-4-2-2-2-B & YP4 & \\
\hline YTH 65 & IR84635-10-59-4-8-3-3-2-4-B & YP3 & & YTH 127 & IR84643-11-11-1-7-7-2-4-2-2-B & YP11 & \\
\hline YTH 66 & IR84635-10-79-9-2-2-3-2-2-B & YP3 & & YTH 128 & IR84643-11-1-7-10-4-2-4-2-2-2-B & YP11 & \\
\hline YTH 67 & IR84635-10-79-9-6-2-3-2-2-B & YP3 & $\mathrm{X}$ & YTH 129 & IR84643-11-1-8-7-2-3-2-2-B & YP11 & \\
\hline YTH 68 & IR84635-10-89-3-1-2-2-2-B & YP3 & $\mathrm{X}$ & YTH 130 & IR84643-11-1-8-8-2-2-4-2-2-2-B & YP11 & \\
\hline YTH 69 & IR84635-10-89-3-61-2-4-2-2-9-B & YP3 & $\mathrm{X}$ & YTH 131 & IR84643-11-13-8-11-2-3-2-2-B & YP11 & \\
\hline YTH 70 & IR84635-10-90-2-9-3-2-4-2-2-2-B & YP3 & & YTH 132 & IR84643-11-15-6-2-3-2-4-3-2-2-B & YP11 & \\
\hline YTH 71 & IR84635-10-90-2-11-2-2-4-2-2-2-B & YP3 & & YTH 133 & IR84643-11-15-6-5-3-2-4-3-2-2-B & YP11 & $\mathrm{X}$ \\
\hline YTH 72 & IR84635-10-91-3-1-3-4-2-2-3-B & YP3 & $\mathrm{X}$ & YTH 134 & IR84643-11-23-8-2-2-3-2-2-B & YP11 & \\
\hline YTH 73 & IR84635-10-91-5-1-3-2-2-B & YP3 & $\mathrm{X}$ & YTH 135 & IR84643-11-23-8-8-2-2-4-2-2-4-B & YP11 & $\mathrm{X}$ \\
\hline YTH 74 & IR84635-10-99-7-4-3-6-2-2-B & YP3 & $\mathrm{X}$ & YTH 136 & IR84643-11-47-6-2-2-2-4-2-2-3-B & YP11 & \\
\hline YTH 75 & IR84635-10-99-7-11-3-3-2-5-B & YP3 & & YTH 137 & IR84643-11-47-6-11-2-2-4-2-2-4-B & YP11 & $\mathrm{X}$ \\
\hline YTH 76 & IR84635-10-111-3-10-1-2-2-2-B & YP3 & & YTH 138 & IR84643-11-68-9-3-2-2-4-3-2-14-B & YP11 & \\
\hline YTH 77 & IR84635-10-113-11-26-3-2-2-B & YP3 & $\mathrm{X}$ & YTH 139 & IR84643-11-68-9-6-2-3-2-2-B & YP11 & \\
\hline YTH 78 & IR84635-10-113-11-98-2-4-2-2-3-B & YP3 & & YTH 140 & IR84643-11-68-11-6-2-3-2-3-B & YP11 & \\
\hline
\end{tabular}


Table 2. Continued.

\begin{tabular}{|c|c|c|c|c|c|c|c|}
\hline $\begin{array}{l}\text { Entry No. } \\
\text { in } 2005 \mathrm{WS}\end{array}$ & IR designation number & $\begin{array}{l}\text { Donor } \\
\text { parent }\end{array}$ & $\begin{array}{c}\text { Core } \\
\text { set }\end{array}$ & $\begin{array}{l}\text { Entry No. } \\
\text { in } 2005 \mathrm{WS}\end{array}$ & IR designation number & $\begin{array}{l}\text { Donor } \\
\text { parent }\end{array}$ & $\begin{array}{c}\text { Core } \\
\text { set }\end{array}$ \\
\hline YTH 142 & IR84643-11-68-11-8-3-2-4-2-2-3-B & YP11 & & YTH 205 & IR84636-13-78-11-3-2-2-4-2-2-2-B & YP5 & $\mathrm{X}$ \\
\hline YTH 143 & IR84643-11-69-3-6-2-2-4-2-2-2-B & YP11 & & YTH 206 & IR84636-13-78-11-8-2-5-4-2-2-2-B & YP5 & \\
\hline YTH 144 & IR84643-11-69-3-9-2-3-2-2-B & YP11 & & YTH 207 & IR84636-13-80-4-4-2-2-4-2-2-2-B & YP5 & \\
\hline YTH 145 & IR84643-11-74-3-2-3-3-2-2-B & YP11 & & YTH 208 & IR84636-13-80-4-10-2-3-2-2-B & YP5 & \\
\hline YTH 146 & IR84643-11-81-4-4-3-3-2-4-B & YP11 & $\mathrm{X}$ & YTH 209 & IR84636-13-80-5-3-2-3-4-2-2-2-B & YP5 & \\
\hline YTH 147 & IR84643-11-81-4-6-3-2-4-2-2-2-B & YP11 & & YTH 210 & IR84636-13-80-5-4-2-2-4-2-2-3-B & YP5 & $X$ \\
\hline YTH 148 & IR84643-11-82-8-2-3-3-2-3-B & YP11 & & YTH 211 & IR84636-13-80-9-9-4-4-2-2-B & YP5 & \\
\hline YTH 149 & IR84643-11-82-8-9-3-2-4-2-2-2-B & YP11 & & YTH 212 & IR84636-13-82-2-2-1-2-4-2-2-2-B & YP5 & \\
\hline YTH 150 & IR84643-11-105-5-5-2-3-2-6-8-B & YP11 & $X$ & YTH 213 & IR84636-13-82-2-5-2-2-4-2-2-2-B & YP5 & $X$ \\
\hline YTH 151 & IR84643-11-105-5-8-3-3-2-2-B & YP11 & & YTH 214 & IR84636-13-82-3-2-3-2-4-2-2-2-B & YP5 & \\
\hline YTH 152 & IR84643-11-105-7-2-2-3-3-3-B & YP11 & & YTH 215 & IR84636-13-82-3-7-3-2-4-2-2-2-B & YP5 & \\
\hline YTH 153 & IR84643-11-105-7-9-2-3-2-2-B & YP11 & $X$ & YTH 216 & IR84636-13-82-12-6-3-2-4-2-2-2-B & YP5 & \\
\hline YTH 154 & IR84643-11-105-8-3-2-2-4-2-3-3-B & YP11 & & YTH 217 & IR84636-13-82-12-8-3-2-4-2-2-3-B & YP5 & \\
\hline YTH 155 & IR84643-11-105-8-7-2-2-4-3-2-3-B & YP11 & $X$ & YTH 218 & IR84636-13-89-11-3-2-2-4-2-2-2-B & YP5 & $X$ \\
\hline YTH 156 & IR84643-11-105-11-6-1-2-4-2-2-3-B & YP11 & & YTH 219 & IR84636-13-89-11-7-2-2-4-2-2-3-B & YP5 & \\
\hline YTH 157 & IR84643-11-105-11-11-3-2-4-2-2-2-B & YP11 & $\mathrm{X}$ & YTH 220 & IR84636-13-90-2-2-2-2-4-2-2-4-B & YP5 & \\
\hline YTH 158 & IR84643-11-106-3-3-2-1-2-3-B & YP11 & & YTH 222 & IR84636-13-90-2-9-2-2-4-2-2-8-B & YP5 & $\mathrm{X}$ \\
\hline YTH 159 & IR84643-11-106-3-10-2-2-2-2-B & YP11 & & YTH 223 & IR84636-13-90-2-10-2-2-4-2-2-5-B & YP5 & \\
\hline YTH 160 & IR84643-11-106-4-6-2-1-3-4-B & YP11 & $\mathrm{X}$ & YTH 224 & IR84636-13-91-3-6-1-2-4-2-2-4-B & YP5 & \\
\hline YTH 162 & IR84643-11-106-4-8-2-8-4-2-2-2-B & YP11 & $\mathrm{X}$ & YTH 225 & IR84636-13-91-3-10-1-2-4-2-2-10-B & YP5 & $X$ \\
\hline YTH 163 & IR84643-11-114-5-7-2-6-2-2-B & YP11 & & YTH 226 & IR84636-13-91-8-4-1-2-4-2-2-5-B & YP5 & $\mathrm{X}$ \\
\hline YTH 164 & IR84643-11-114-5-9-2-3-4-2-2-2-B & YP11 & & YTH 227 & IR84636-13-91-8-8-1-2-4-2-2-2-B & YP5 & \\
\hline YTH 165 & IR84643-11-114-6-2-2-3-2-2-B & YP11 & $\mathrm{X}$ & YTH 229 & IR84637-4-7-8-2-2-3-4-3-2-2-B & YP6 & \\
\hline YTH 166 & IR84643-11-114-6-3-2-4-4-3-2-2-B & YP11 & & YTH 230 & IR84637-4-7-10-3-2-2-4-2-2-2-B & YP6 & \\
\hline YTH 167 & IR84643-11-115-4-2-3-2-5-2-2-2-B & YP11 & & YTH 231 & IR84637-4-7-10-8-3-3-4-4-2-2-B & YP6 & \\
\hline YTH 168 & IR84643-11-115-4-5-3-2-4-2-2-2-B & YP11 & & YTH 232 & IR84637-4-7-10-9-2-2-4-2-2-2-B & YP6 & \\
\hline YTH 169 & IR84636-13-2-2-3-2-2-4-3-2-2-B & YP5 & & YTH 233 & IR84637-4-29-5-2-3-1-2-2-B & YP6 & \\
\hline YTH 170 & IR84636-13-2-2-9-2-2-4-2-2-2-B & YP5 & & YTH 234 & IR84637-4-29-5-7-2-2-4-2-2-4-B & YP6 & \\
\hline YTH 171 & IR84636-13-2-3-2-2-2-4-2-2-2-B & YP5 & & YTH 235 & IR84637-4-63-8-3-1-2-5-2-2-2-B & YP6 & \\
\hline YTH 172 & IR84636-13-2-3-5-2-3-3-6-B & YP5 & & YTH 236 & IR84637-4-63-8-8-2-3-2-2-B & YP6 & \\
\hline YTH 173 & IR84636-13-5-2-2-2-3-2-3-B & YP5 & & YTH 237 & IR84637-4-63-8-11-2-2-4-2-2-2-B & YP6 & \\
\hline YTH 174 & IR84636-13-5-2-9-2-2-4-2-2-2-B & YP5 & $\mathrm{X}$ & YTH 238 & IR84637-4-63-11-4-2-2-4-2-2-2-B & YP6 & \\
\hline YTH 175 & IR84636-13-5-7-2-2-3-2-3-B & YP5 & & YTH 239 & IR84637-4-63-11-10-2-3-2-2-B & YP6 & \\
\hline YTH 176 & IR84636-13-5-7-7-2-3-2-2-B & YP5 & & YTH 240 & IR84637-4-69-3-2-3-3-2-3-B & YP6 & \\
\hline YTH 177 & IR84636-13-8-5-4-2-3-2-2-B & YP5 & & YTH 242 & IR84637-4-69-3-5-1-3-4-2-2-2-B & YP6 & \\
\hline YTH 178 & IR84636-13-8-5-8-2-2-4-2-2-3-B & YP5 & & YTH 243 & IR84637-4-69-7-2-2-2-4-2-2-2-B & YP6 & $\mathrm{X}$ \\
\hline YTH 179 & IR84636-13-8-6-5-2-2-4-2-2-8-B & YP5 & & YTH 244 & IR84637-4-69-7--10-1-2-4-2-2-2-B & YP6 & \\
\hline YTH 180 & IR84636-13-8-6-7-2-2-4-2-2-2-B & YP5 & $\mathrm{X}$ & YTH 245 & IR84b37-4-69-9-6-1-4-2-2-B & YP6 & $\mathrm{X}$ \\
\hline YTH 182 & IR84636-13-12-2-2-2-2-4-2-2-2-B & YP5 & & YTH 246 & IR84637-4-69-9-10-2-4-4-2-2-2-B & YP6 & $\mathrm{X}$ \\
\hline YTH 183 & IR84636-13-12-2-6-3-3-2-2-B & YP5 & $\mathrm{X}$ & YTH 247 & IR84637-4-69-12-3-3-3-2-3-B & YP6 & $\mathrm{X}$ \\
\hline YTH 184 & IR84636-13-12-3-5-2-2-4-2-3-3-B & YP5 & & YTH 248 & IR84637-4-69-12-10-3-2-4-4-2-2-B & YP6 & \\
\hline YTH 185 & IR84636-13-12-3-9-2-2-4-2-2-2-B & YP5 & & YTH 249 & IR84637-4-72-9-4-1-2-4-2-2-2-B & YP6 & \\
\hline YTH 186 & IR84636-13-53-5-7-2-2-4-2-2-2-B & YP5 & $\mathrm{X}$ & YTH 250 & IR84637-4-72-9-5-2-2-4-2-2-2-B & YP6 & \\
\hline YTH 187 & IR84636-13-55-5-8-2-2-4-2-2-2-B & YP5 & $\mathrm{X}$ & YTH 251 & IR84637-4-72-9-10-1-2-4-2-2-2-B & YP6 & \\
\hline YTH 188 & IR84636-13-55-8-5-2-3-2-7-B & YP5 & & YTH 252 & IR84637-4-74-2-2-2-3-2-2-B & YP6 & $\mathrm{X}$ \\
\hline YTH 189 & IR84636-13-55-8-11-2-2-4-2-2-3-B & YP5 & & YTH 253 & IR84637-4-74-2-7-2-3-2-3-B & YP6 & \\
\hline YTH 190 & IR84636-13-59-6-3-2-3-4-5-2-2-B & YP5 & & YTH 254 & IR84637-4-89-2-9-2-3-2-2-B & YP6 & $\mathrm{X}$ \\
\hline YTH 191 & IR84636-13-59-6-4-2-2-4-2-2-2-B & YP5 & $\mathrm{X}$ & YTH 255 & IR84637-4-106-7-11-2-2-2-2-B & YP6 & $\mathrm{X}$ \\
\hline YTH 192 & IR84636-13-64-2-4-2-2-4-2-2-3-B & YP5 & & YTH 256 & IR84637-4-113-6-6-2-2-Z-7-B & YP6 & \\
\hline YTH 193 & IR84636-13-64-2-5-2-2-4-2-2-7-B & YP5 & & YTH 257 & IR84637-4-113-6-10-2-3-2-14-B & YP6 & \\
\hline YTH 194 & IR84636-13-71-7-3-2-3-2-3-B & YP5 & & YTH 258 & IR84637-4-113-11-5-2-3-2-2-B & YP6 & \\
\hline YTH 195 & IR84636-13-71-7-5-2-2-4-2-2-2-B & YP5 & & YTH 259 & IR84638-11-6-3-2-1-1-2-2-B & YP7 & $\mathrm{X}$ \\
\hline YTH 196 & IR84636-13-71-9-2-3-2-4-2-2-2-B & YP5 & $\mathrm{X}$ & YTH 260 & IR84638-11-6-3-7-1-2-5-4-2-2-B & YP7 & $\mathrm{X}$ \\
\hline YTH 197 & IR84636-13-71-9-10-3-4-2-2-B & YP5 & $\mathrm{X}$ & YTH 262 & IR84638-11-11-9-2-1-2-4-2-2-2-B & YP7 & $\mathrm{X}$ \\
\hline YTH 198 & IR84636-13-71-10-2-2-2-4-3-2-14-B & YP5 & $\mathrm{X}$ & YTH 263 & IR84638-11-11-9-7-1-2-4-2-2-2-B & YP7 & \\
\hline YTH 199 & IR84636-13-71-11-3-2-2-4-2-2-2-B & YP5 & $\mathrm{X}$ & YTH 264 & IR84638-11-11-9-9-1-2-5-2-2-2-B & YP7 & $\mathrm{X}$ \\
\hline YTH 200 & IR84636-13-71-11-4-2-2-4-2-2-2-B & YP5 & $\mathrm{X}$ & YTH 265 & IR84638-11-17-10-4-1-2-4-2-2-2-B & YP7 & \\
\hline YTH 202 & IR84636-13-71-11-7-2-3-2-2-B & YP5 & & YTH 266 & IR84638-11-17-10-9-1-2-4-2-2-2-B & YP7 & $\mathrm{X}$ \\
\hline YTH 203 & IR84636-13-78-9-4-2-2-4-2-2-2-B & YP5 & $X$ & YTH 267 & IR84638-11-60-6-3-1-2-4-2-2-2-B & YP7 & \\
\hline YTH 204 & IR84636-13-78-9-5-2-2-4-2-2-5-B & YP5 & & YTH 268 & IR84638-11-60-6-10-1-2-4-2-2-2-B & YP7 & \\
\hline
\end{tabular}


D. Fujita et al.

Table 2. Continued.

\begin{tabular}{|c|c|c|c|}
\hline $\begin{array}{l}\text { Entry No. } \\
\text { in 2005WS }\end{array}$ & IR designation number & $\begin{array}{l}\text { Donor } \\
\text { parent }\end{array}$ & $\begin{array}{c}\text { Core } \\
\text { set }\end{array}$ \\
\hline YTH 269 & IR84638-11-66-8-3-1-2-4-2-2-2-B & YP7 & $\mathrm{X}$ \\
\hline YTH 270 & IR84638-11-66-8-4-1-2-4-2-2-2-B & YP7 & $\mathrm{X}$ \\
\hline YTH 271 & IR84638-11-66-8-7-1-2-4-4-2-2-B & YP7 & $\mathrm{X}$ \\
\hline YTH 272 & IR84638-11-85-2-5-1-3-2-2-B & YP7 & $X$ \\
\hline YTH 273 & IR84638-11-85-2-7-1-2-4-3-2-2-B & YP7 & \\
\hline YTH 274 & IR84638-11-101-5-4-1-4-2-2-B & YP7 & \\
\hline YTH 275 & IR84638-11-101-5-9-3-2-4-2-2-2-B & YP7 & \\
\hline YTH 276 & IR84638-11-101-5-10-4-2-4-2-2-2-B & YP7 & \\
\hline YTH 277 & IR84638-11-101-9-6-1-2-4-3-2-2-B & YP7 & $\mathrm{X}$ \\
\hline YTH 278 & IR84638-11-101-9-8-1-2-4-2-2-2-B & YP7 & \\
\hline YTH 279 & IR84638-11-101-9-9-3-2-4-2-2-2-B & YP7 & $\mathrm{X}$ \\
\hline YTH 280 & IR84638-11-118-8-10-3-2-4-2-2-2-B & YP7 & \\
\hline YTH 282 & IR84639-7-12-4-3-1-2-4-2-2-2-B & YP8 & \\
\hline YTH 283 & IR84639-7-12-4-3-1-2-4-2-2-2-B & YP8 & \\
\hline YTH 284 & IR84639-7-21-9-3-3-2-4-2-2-2-B & YP8 & $\mathrm{X}$ \\
\hline YTH 285 & IR84639-7-21-9-8-3-3-2-5-B & YP8 & \\
\hline YTH 286 & IR84639-7-21-9-9-2-2-4-2-2-2-B & YP8 & \\
\hline YTH 287 & IR84639-7-28-5-4-2-2-4-3-2-2-B & YP8 & $\mathrm{X}$ \\
\hline YTH 288 & IR84639-7-28-5-5-2-2-3-2-2-14-B & YP8 & $\mathrm{X}$ \\
\hline YTH 289 & IR84639-7-29-3-8-3-2-4-2-2-3-B & YP8 & $\mathrm{X}$ \\
\hline YTH 290 & IR84639-7-29-3-10-2-2-2-3-B & YP8 & \\
\hline YTH 291 & IR84639-7-65-1-5-2-2-4-2-2-3-B & YP8 & \\
\hline YTH 292 & IR84639-7-65-1-11-3-2-4-2-2-7-B & YP8 & $\mathrm{X}$ \\
\hline YTH 293 & IR84639-7-72-5-2-3-2-4-2-2-2-B & YP8 & \\
\hline YTH 294 & IR84639-7-72-5-9-2-3-4-2-2-3-B & YP8 & \\
\hline YTH 295 & IR84639-7-72-8-3-1-3-2-2-B & YP8 & \\
\hline YTH 296 & IR84639-7-72-8-4-2-2-4-2-2-2-B & YP8 & \\
\hline YTH 297 & IR84639-7-76-3-2-1-2-4-2-2-2-B & YP8 & $\mathrm{X}$ \\
\hline YTH 298 & IR84639-7-76-3-5-1-2-4-3-2-2-B & YP8 & \\
\hline YTH 299 & IR84639-7-76-8-3-3-2-4-2-2-2-B & YP8 & \\
\hline YTH 300 & IR84639-7-76-8-6-3-3-2-2-B & YP8 & \\
\hline YTH 302 & IR84639-7-88-5-6-3-3-2-3-B & YP8 & $\mathrm{X}$ \\
\hline YTH 303 & IR84639-7-88-5-11-5-2-5-3-2-2-B & YP8 & $\mathrm{X}$ \\
\hline YTH 304 & IR84639-7-97-6-6-2-2-4-2-2-2-B & YP8 & $\mathrm{X}$ \\
\hline YTH 305 & IR84639-7-97-7-4-3-2-4-2-2-3-B & YP8 & \\
\hline YTH 306 & IR84639-7-110-2-3-2-2-4-2-2-2-B & YP8 & $\mathrm{X}$ \\
\hline YTH 307 & IR84639-7-110-2-7-1-4-2-4-B & YP8 & \\
\hline YTH 308 & IR84639-7-110-3-4-2-3-4-2-2-2-B & YP8 & \\
\hline YTH 309 & IR84639-7-110-3-7-1-3-2-2-B & YP8 & \\
\hline YTH 310 & IR84639-7-115-4-5-2-3-2-2-B & YP8 & \\
\hline YTH 311 & IR84639-7-115-4-6-3-3-2-2-B & YP8 & \\
\hline YTH 312 & IR84640-11-3-1-5-2-2-4-2-2-2-B & YP9 & \\
\hline YTH 313 & IR84640-11-3-1-6-2-3-2-5-B & YP9 & \\
\hline YTH 314 & IR84640-11-3-6-7-2-2-4-2-2-5-B & YP9 & \\
\hline YTH 315 & IR84640-11-3-6-8-1-3-2-3-B & YP9 & \\
\hline YTH 316 & IR84640-11-10-7-4-2-2-4-2-3-2-3-B & YP9 & \\
\hline YTH 317 & IR84640-11-10-7-7-2-2-4-2-2-2-B & YP9 & \\
\hline YTH 318 & IR84640-11-12-7-4-2-2-4-2-3-2-3-B & YP9 & $\mathrm{X}$ \\
\hline YTH 319 & IR84640-11-14-10-3-2-2-4-2-2-2-B & YP9 & $\mathrm{X}$ \\
\hline YTH 320 & IR84640-11-14-10-9-2-2-4-2-2-2-B & YP9 & \\
\hline YTH 322 & IR84640-11-27-1-5-3-3-2-2-B & YP9 & \\
\hline YTH 323 & IR84640-11-27-1-9-3-2-4-2-2-2-B & YP9 & $\mathrm{X}$ \\
\hline YTH 324 & IR84640-11-37-1-6-3-3-4-2-2-2-B & YP9 & $\mathrm{X}$ \\
\hline YTH 325 & IR84640-11-110-6-2-2-2-4-2-2-4-B & YP9 & $\mathrm{X}$ \\
\hline YTH 326 & IR84640-11-110-6-4-2-2-4-2-2-3-B & YP9 & $\mathrm{X}$ \\
\hline YTH 327 & IR84640-11-110-7-2-2-2-2-2-B & YP9 & \\
\hline YTH 328 & IR84640-11-110-7-9-2-2-5-3-2-2-B & YP9 & $\mathrm{X}$ \\
\hline
\end{tabular}

\begin{tabular}{|c|c|c|c|}
\hline $\begin{array}{l}\text { Entry No. } \\
\text { in } 2005 \mathrm{WS}\end{array}$ & IR designation number & $\begin{array}{l}\text { Donor } \\
\text { parent }\end{array}$ & $\begin{array}{c}\text { Core } \\
\text { set }\end{array}$ \\
\hline YTH 329 & IR84641-2-15-2-3-2-2-4-2-2-2-B & YP10 & \\
\hline YTH 330 & IR84641-2-22-2-10-2-2-4-3-2-2-B & YP10 & \\
\hline YTH 331 & IR84641-2-61-I1-7-2-2-4-2-2-2-B & YP10 & \\
\hline YTH 332 & IR84641-2-71-5-11-2-3-2-2-B & YP10 & $\mathrm{X}$ \\
\hline YTH 333 & IR84641-2-76-2-9-1-2-4-2-2-2-B & YP10 & \\
\hline YTH 334 & IR84641-2-76-4-2-2-2-4-4-2-2-B & YP10 & \\
\hline YTH 335 & IR84641-2-76-4-4-2-3-2-2-B & YP10 & \\
\hline YTH 336 & IR84641-6-5-2-2-2-3-2-2-B & YP10 & $\mathrm{X}$ \\
\hline YTH 337 & IR84641-6-5-2-3-2-2-4-2-2-2-B & YP10 & \\
\hline YTH 338 & IR84641-6-5-2-6-1-2-4-2-2-2-B & YP10 & \\
\hline YТН 339 & IR84641-6-12-6-2-3-3-2-2-B & YP10 & $\mathrm{X}$ \\
\hline YTH 340 & IR84641-6-12-6-6-2-3-2-2-B & YP10 & \\
\hline YTH 342 & IR84641-6-12-8-2-2-2-4-2-2-2-B & YP10 & $\mathrm{X}$ \\
\hline YTH 343 & IR84641-6-12-8-10-1-2-4-2-2-2-B & YP10 & \\
\hline YTH 344 & IR84641-6-17-5-7-2-2-4-2-2-2-B & YP10 & $\mathrm{X}$ \\
\hline YTH 345 & IR84641-6-23-11-3-1-2-4-2-2-2-B & YP10 & \\
\hline YTH 346 & IR84641-6-23-11-10-2-2-4-2-2-2-B & YP10 & \\
\hline YTH 347 & IR84641-6-30-5-11-2-3-2-3-B & YP10 & \\
\hline YTH 348 & IR84641-6-39-3-3-2-2-4-2-2-3-B & YP10 & \\
\hline YTH 349 & IR84641-6-39-3-9-1-3-2-2-B & YP10 & \\
\hline YTH 350 & IR84641-6-46-10-2-2-3-2-2-B & YP10 & \\
\hline YTH 351 & IR84641-6-63-2-3-1-3-2-3-B & YP10 & $\mathrm{X}$ \\
\hline YTH 352 & IR84641-6-63-2-9-4-3-2-2-B & YP10 & \\
\hline YTH 353 & IR84641-6-63-2-11-3-3-2-2-B & YP10 & $\mathrm{X}$ \\
\hline YTH 354 & IR84641-6-66-3-2-1-2-2-2-B & YP10 & \\
\hline YTH 355 & IR84641-6-66-3-11-1-3-2-2-B & YP10 & \\
\hline YTH 356 & IR84641-6-84-8-2-3-3-2-2-B & YP10 & \\
\hline YTH 357 & IR84641-6-84-8-3-3-3-2-2-B & YP10 & \\
\hline YTH 358 & IR84641-6-85-8-11-5-3-2-3-B & YP10 & \\
\hline YTH 359 & IR84641-6-87-2-7-1-3-2-2-B & YP10 & \\
\hline YTH 360 & IR84641-6-87-2-10-1-2-4-2-2-2-B & YP10 & $\mathrm{X}$ \\
\hline YTH 362 & IR84641-6-96-3-3-2-2-2-2-B & YP10 & $\mathrm{X}$ \\
\hline YTH 363 & IR84641-6-111-5-4-2-2-2-2-B & YP10 & $\mathrm{X}$ \\
\hline YTH 364 & IR84641-6-111-5-9-1-3-2-2-B & YP10 & \\
\hline YTH 365 & IR84641-6-111-5-10-3-3-2-2-B & YP10 & \\
\hline YTH 366 & IR84641-6-116-2-8-3-3-2-2-B & YP10 & \\
\hline YTH 367 & IR84641-6-116-2-10-1-3-2-2-B & YP10 & \\
\hline YTH 368 & IR84641-6-119-9-2-2-4-4-4-B & YP10 & $\mathrm{X}$ \\
\hline YTH 369 & IR84641-6-119-9-7-2-3-2-2-B & YP10 & \\
\hline
\end{tabular}

a) see Table 1 
each of $95^{\circ} \mathrm{C}$ for $30 \mathrm{~s}, 55^{\circ} \mathrm{C}$ for $30 \mathrm{~s}$, and $72^{\circ} \mathrm{C}$ for 30 $\mathrm{s}$. The SSR products were resolved in $4.0 \%$ agarose gel by electrophoresis at $250 \mathrm{~V}$ for $1 \mathrm{~h}$ in $0.5 \times \mathrm{TBE}$ buffer. The gels were stained with ethidium bromide and photographed under ultraviolet light.

\section{Results}

\section{Characterization of INLs in agronomic traits}

The 334 INLs for agronomic traits from 10 donor varieties were developed by backcrossed breeding and used for association analysis between introgressed segments and agronomic traits (Table 2). The eight agronomic traits: DTH, CL, LW, LL, PL, PN, TSN, and GW in the 334 INLs were evaluated from 2005 to 2007. Based on the data obtained in WS and DS, a total of 117 INLs were selected as a 'core set' for further detailed characterization. The unique INLs that showed the top 30 and the least 30 values on six agronomic traits: DTH, CL, LW, LL, PL, and PN in 2005WS, 2006DS, 2006WS, and 2007DS were selected. Some unique INLs showed a similar phenotype and genotype to each other. In this case, a single line was selected among these INLs to avoid duplication. The core set consisted of 10 lines from YP1, 12 lines from YP3, 19 lines from YP4, 19 lines from YP5, seven lines from YP6, 11 lines from YP7, 10 lines from YP8, seven lines from YP9, 11 lines from YP10, and 11 lines from YP11 (Tables 1 and 2). The selected 117 INLs covered the variations of agronomic traits: DTH, CL, LW, LL, PL, PN, TSN, and GW, observed in the developed 334 INLs (Fig. 1).

On the selected 117 lines, eight agronomic traits: DTH, CL, PL, LW, LL, PN, TSN, and GW, were evaluated from 2005 to 2008 (Table 3). Some of the INLs among the same donor parents showed unique agronomic traits compared with IR64. For DTH, several INLs from YP1, YP7 and YP10 were shorter than IR64, while several lines from YP3, YP5, YP6, and YP8 were longer than IR64. For CL, several INLs from YP1, YP5, YP7, and YP10 were shorter than IR64, while several INLs from YP3, YP4, YP8, YP9, and YP11 were longer. For PL, several INLs from YP1, YP7 and YP10 were shorter than IR64, while several INLs from YP3 and YP 4 were longer. For LW, several INLs from YP1, YP5 and YP10 had a narrow leaf compared with IR64, while several INLs from YP3, YP4, YP8, YP9, and YP11 had a wider leaf. LL of several INLs from YP1, YP5 and YP 10 were shorter than that of IR64, while that of several INLs from YP3, YP4, YP8, YP9, and YP11 were longer. PN of several INLs from YP7 were higher than that of IR64, while that of several INLs from YP3 and YP4 were lower. TSN of several INLs from YP1, YP7 and
YP10 were lower than that of IR64, while that of several INLs from YP3, YP4, YP5, YP7, YP8, YP9, and YP 11 were higher. For GW, several INLs from YP1, YP5 and YP10 had heavier grain than IR64. The 117 INLs which showed variations in agronomic traits of DTH, CL, PL, LL, LW, PN, TSN, and GW, had different characteristics depending on donor varieties.

\section{Determination of genotypes in the 334 INLs}

The genotypes of the 334 INLs were analyzed using the polymorphic SSR markers (those of the selected 117 INLs are shown in Fig. 2). The common regions of introgressed segment for each donor parent were found on chromosomes 1, 2, 4, 5, and 6 among more than four sib groups. Twenty-nine lines out of them from YP 4, YP5 and YP11, had the introgressed segments at the region around 130 to $140 \mathrm{cM}$ on the long arm of chromosome 1. Forty-two lines from YP1, YP5, YP6, YP8, YP9, and YP10 had the introgressed segments at from 0 to $10 \mathrm{cM}$ on chromosome 2. Forty-five lines from YP1, YP4, YP5, YP8, YP9, and YP11 had the introgressed segments at from 110 to $120 \mathrm{cM}$ on chromosome 4. Thirty-five lines from YP1, YP3, YP5, YP9, YP10, and YP11 had the introgressed segments at from 40 to 60 $\mathrm{cM}$ on chromosome 5. Twenty-six lines from YP5, YP6, YP7, and YP10 had the introgressed segments at from 80 to $90 \mathrm{cM}$ on chromosome 6 .

\section{Comparisons of DTH, TSN and GW between the INLs and IR64}

Association analysis between genotype and agronomic traits in 334 INLs was conducted in a previous study and 54 associated regions for agronomic traits in nine sib groups were detected by analysis of variation ${ }^{2}$. Several associated regions for DTH, GW and TSN were located on chromosomes 1, 4, 5, 6, 8, and 12 (Fig. 3). Representative INLs showed significant difference in these traits with IR64 in 2007 WS and 2008 DS (Fig. 4). Most of these INLs had associated regions for DTH, GW and TSN, while several other lines didn't have associated regions for DTH, GW and TSN. DTH of two INLs in DS were shorter than that of IR64: by 10 days in YTH13 (derived from YP1) and by 11 days in YTH 259 (YP7) (Fig. 4A). DTH of two INLs in DS were longer than that of IR64: by 22 days longer in YTH59 (YP3) and by eight days longer in YTH246 (YP6). Three INLs for DTH had associated regions: YTH259 with YP7-DTH12 for short DTH, YTH13 with YP1DTH8 for short DTH and YTH59 with YP3-DTH6 for long DTH. The name for an associated region, for example, YP7-DTH12, represented the donor parent YP7, followed by the trait DTH and chromosome number 12 . 

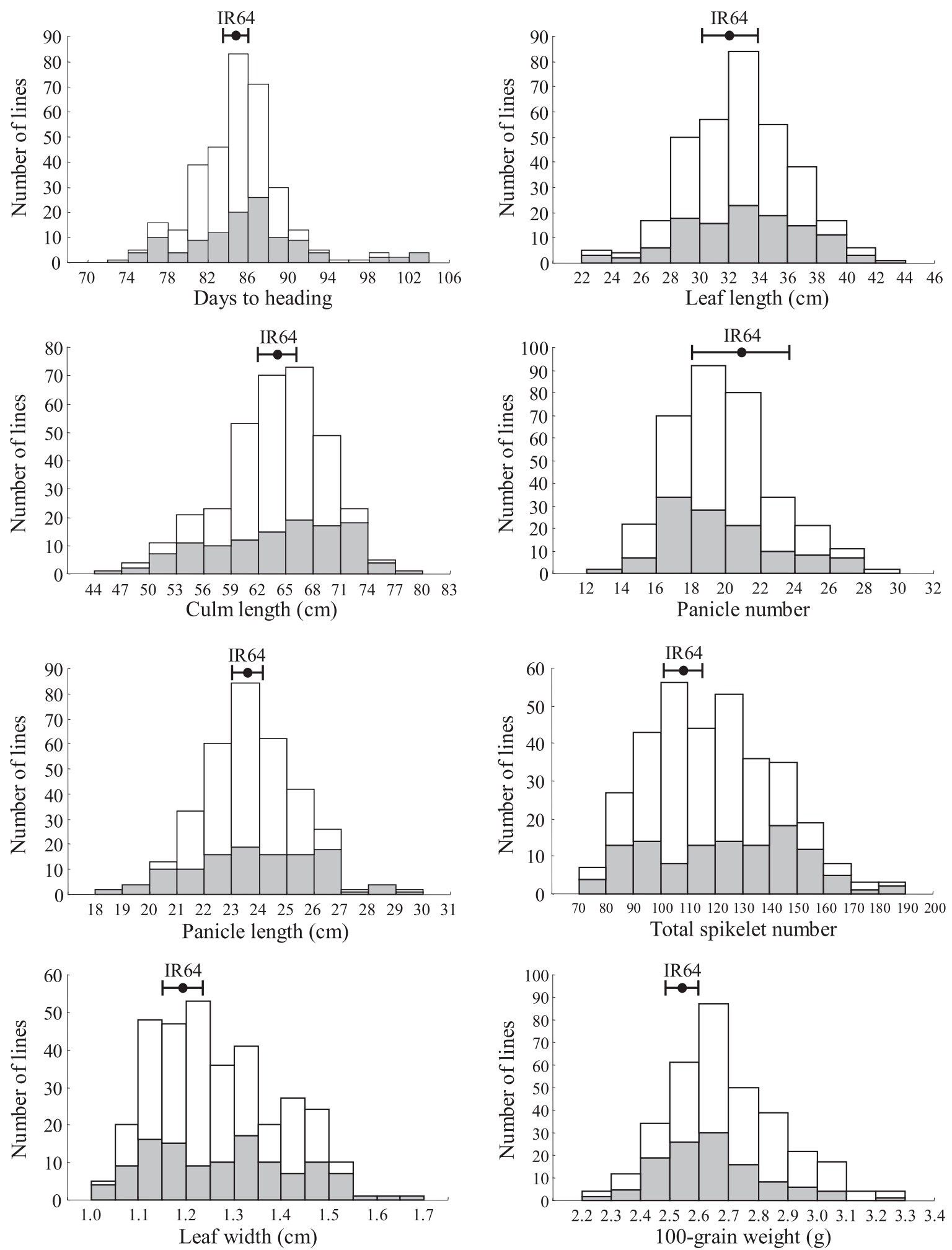

Fig. 1. Frequency distribution of eight agronomic traits: days to heading, culm length, panicle length, leaf width, leaf length, panicle number, total spikelet number per panicle, and 100-grain weight in 334 introgression lines at dry season

Gray area indicates 117 introgression lines, while white area indicates the remaining 217 lines in each trait. Error bar under IR64 showed standard deviation of IR64. 
Table 3. Variation in agronomic traits of 117 introgression lines bred from the crosses between IR64 as a recurrent variety and 10 different donor varieties.

\begin{tabular}{|c|c|c|c|c|c|c|c|c|c|c|c|c|c|c|c|c|c|}
\hline \multirow{3}{*}{$\begin{array}{l}\text { 2005WS } \\
\text { Entry No. }\end{array}$} & \multirow{3}{*}{$\begin{array}{l}\text { Donor } \\
\text { variety }\end{array}$} & \multicolumn{16}{|c|}{ Average of agronomic traits in each INL } \\
\hline & & \multicolumn{2}{|c|}{ DTH(day) } & \multicolumn{2}{|c|}{$\mathrm{CL}(\mathrm{cm})$} & \multicolumn{2}{|c|}{ PL $(\mathrm{cm})$} & LW & $\mathrm{cm})$ & LL( & $\mathrm{cm})$ & $\mathrm{P}$ & $\mathrm{N}$ & TS & & & \\
\hline & & DS & WS & DS & WS & DS & WS & DS & WS & DS & WS & DS & WS & DS & WS & DS & WS \\
\hline IR64 & & 86 & 90 & 63.5 & 64.8 & 24.0 & 24.2 & 1.2 & 1.2 & 34.4 & 33.0 & 21.0 & 18.2 & 108.8 & 104.7 & 2.6 & 2.4 \\
\hline YP1 & & 104 & 93 & 74.1 & 70.9 & 25.6 & 25.0 & 1.9 & 1.9 & 40.2 & 39.9 & 10.2 & 10.8 & 154.6 & 142.2 & 3.7 & 3.7 \\
\hline YTH 6 & YP1 & 78 & 88 & 54.0 & 68.8 & 20.6 & 21.9 & 1.1 & 1.3 & 28.2 & 35.4 & 22.1 & 19.6 & 84.7 & 92.0 & 2.8 & 2.9 \\
\hline YTH 10 & YP1 & 79 & 83 & 57.7 & 66.1 & 22.6 & 23.7 & 1.1 & 1.2 & 32.5 & 37.3 & 22.6 & 19.4 & 84.7 & 83.4 & 2.7 & 2.5 \\
\hline YTH 12 & YP1 & 76 & 80 & 57.9 & 66.3 & 20.6 & 22.2 & 1.1 & 1.1 & 29.1 & 34.2 & 25.8 & 19.8 & 69.7 & 68.6 & 2.7 & 2.5 \\
\hline YTH 13 & YP1 & 76 & 82 & 59.0 & 68.7 & 20.7 & 21.7 & 1.1 & 1.1 & 29.2 & 34.3 & 24.5 & 19.0 & 69.8 & 75.8 & 2.7 & 2.6 \\
\hline YTH 15 & YP1 & 84 & 91 & 69.2 & 76.6 & 23.5 & 23.9 & 1.2 & 1.2 & 33.1 & 37.2 & 22.4 & 17.2 & 95.0 & 82.0 & 2.7 & 2.6 \\
\hline YTH 16 & YPI & 77 & 81 & 59.1 & 64.4 & 21.9 & 23.6 & 1.1 & 1.2 & 30.0 & 36.1 & 21.2 & 19.3 & 87.6 & 89.2 & 2.6 & 2.5 \\
\hline YTH 20 & YP1 & 77 & 82 & 51.9 & 64.3 & 21.0 & 22.8 & 1.2 & 1.2 & 28.7 & 34.6 & 25.9 & 19.7 & 71.7 & 78.1 & 2.9 & 3.2 \\
\hline YTH 24 & YP1 & 83 & 88 & 61.3 & 74.4 & 21.9 & 22.4 & 1.1 & 1.2 & 29.8 & 33.6 & 23.4 & 19.0 & 83.8 & 82.2 & 2.8 & 2.9 \\
\hline YTH 33 & YP1 & 81 & 89 & 60.3 & 72.3 & 23.7 & 24.8 & 1.0 & 1.2 & 32.5 & 39.0 & 20.9 & 17.3 & 91.5 & 88.6 & 3.0 & 3.0 \\
\hline YTH 35 & YP1 & 77 & 83 & 55.5 & 67.5 & 22.0 & 22.8 & 1.2 & 1.3 & 31.7 & 36.5 & 20.0 & 16.8 & 86.2 & 98.7 & 3.0 & 2.9 \\
\hline YP3 & & 104 & 96 & 61.4 & 62.8 & 22.9 & 24.8 & 2.0 & 2.1 & 37.8 & 40.7 & 10.9 & 7.0 & 207.2 & 252.9 & 2.5 & 2.3 \\
\hline YTH 57 & YP3 & 93 & 98 & 66.1 & 74.1 & 27.0 & 29.3 & 1.3 & 1.4 & 35.4 & 41.0 & 17.8 & 15.2 & 146.0 & 137.4 & 2.5 & 2.5 \\
\hline YTH 58 & YP3 & 106 & 125 & 66.3 & 78.0 & 28.7 & 29.4 & 1.4 & 1.4 & 41.7 & 48.1 & 17.3 & 14.5 & 156.2 & 116.9 & 2.3 & 2.5 \\
\hline YTH 59 & YP3 & 108 & 125 & 65.9 & 76.9 & 29.3 & 29.7 & 1.4 & 1.4 & 43.5 & 47.8 & 152 & 14.4 & 158.4 & 122.8 & 2.3 & 2.4 \\
\hline YTH 60 & YP3 & 105 & 116 & 69.8 & 82.8 & 26.4 & 27.2 & 1.3 & 1.3 & 36.7 & 44.0 & 18.0 & 15.5 & 125.8 & 115.9 & 2.6 & 2.5 \\
\hline YTH 63 & YP3 & 91 & 97 & 65.8 & 78.4 & 27.1 & 28.5 & 1.4 & 1.4 & 36.0 & 43.9 & 17.7 & 14.7 & 166.6 & 142.1 & 2.6 & 2.6 \\
\hline YTH 67 & YP3 & 89 & 94 & 67.7 & 76.8 & 28.1 & 27.8 & 1.4 & 1.4 & 35.1 & 39.2 & 17.2 & 13.7 & 148.2 & 126.7 & 2.6 & 2.5 \\
\hline YTH 68 & YP3 & 107 & 118 & 69.1 & 77.4 & 28.1 & 29.3 & 1.4 & 1.4 & 40.2 & 49.0 & 16.6 & 13.7 & 154.5 & 136.3 & 2.4 & 2.5 \\
\hline ҮTH 69 & YP3 & 103 & 114 & 72.9 & 81.7 & 27.2 & 28.1 & 1.3 & 1.4 & 38.1 & 47.6 & 16.8 & 13.7 & 153.5 & 150.5 & 2.6 & 2.4 \\
\hline YTH 72 & YP3 & 105 & 120 & 66.0 & 79.0 & 28.9 & 29.4 & 1.4 & 1.4 & 38.4 & 48.4 & 18.5 & 16.6 & 150.8 & 131.5 & 2.4 & 2.5 \\
\hline YTH 73 & YP3 & 107 & 120 & 66.8 & 79.2 & 29.0 & 29.0 & 1.3 & 1.3 & 39.9 & 47.3 & 18.0 & 14.4 & 157.5 & 139.2 & 2.5 & 2.5 \\
\hline YTH 74 & YP3 & 92 & 97 & 68.9 & 78.4 & 26.6 & 27.6 & 1.3 & 1.3 & 36.7 & 40.2 & 19.2 & 16.7 & 143.5 & 124.3 & 2.6 & 2.6 \\
\hline YTH 77 & YP3 & 100 & 115 & 69.9 & 82.9 & 26.4 & 27.8 & 1.2 & 1.3 & 36.4 & 44.9 & 18.0 & 16.4 & 131.5 & 132.4 & 2.4 & 2.4 \\
\hline YP4 & & 84 & 90 & 78.8 & 78.7 & 23.8 & 25.2 & 1.5 & 1.6 & 39.7 & 40.7 & 10.3 & 8.6 & 180.5 & 210.9 & 2.6 & 2.7 \\
\hline YTH 83 & YP4 & 90 & 92 & 74.4 & 77.4 & 26.4 & 26.9 & 1.5 & 1.5 & 37.7 & 46.5 & 14.8 & 14.2 & 199.8 & 202.0 & 2.3 & 2.2 \\
\hline YTH 84 & YP4 & 89 & 91 & 74.2 & 75.7 & 25.6 & 25.8 & 1.5 & 1.5 & 40.4 & 44.9 & 17.3 & 14.4 & 176.5 & 170.2 & 2.4 & 2.2 \\
\hline YTH 85 & YP4 & 89 & 90 & 69.7 & 77.2 & 25.4 & 26.0 & 1.3 & 1.3 & 36.8 & 39.4 & 18.0 & 16.9 & 150.6 & 123.1 & 2.6 & 2.7 \\
\hline TH 88 & YP4 & 89 & 92 & 74.5 & 76.4 & 27.0 & 27.9 & 1.5 & 1.4 & 40.4 & 44.2 & 18.0 & 13.7 & 177.5 & 183.2 & 2.4 & 2.3 \\
\hline YTH 89 & YP4 & 88 & 92 & 73.8 & 77.5 & 27.4 & 27.4 & 1.4 & 1.4 & 41.4 & 43.6 & 17.6 & 13.4 & 155.2 & 170.0 & 2.5 & 2.4 \\
\hline YTH 92 & YP4 & 87 & 90 & 74.8 & 80.8 & 24.8 & 25.8 & 1.3 & 1.4 & 37.4 & 41.2 & 19.0 & 16.5 & 135.2 & 122.9 & 2.6 & 2.6 \\
\hline YTH 99 & YP4 & 86 & 89 & 73.1 & 80.7 & 24.7 & 25.1 & 1.3 & 1.5 & 36.9 & 41.3 & 19.2 & 16.6 & 161.5 & 142.0 & 2.4 & 2.4 \\
\hline YTH 105 & YP4 & 87 & 93 & 69.6 & 76.1 & 25.7 & 26.2 & 1.5 & 1.5 & 37.2 & 43.3 & 17.0 & 14.5 & 170.0 & 152.9 & 2.4 & 2.5 \\
\hline TH 107 & YP4 & 89 & 92 & 73.9 & 75.6 & 25.6 & 25.5 & 1.5 & 1.5 & 39.0 & 45.2 & 14.8 & 13.7 & 159.3 & 187.7 & 2.4 & 2.4 \\
\hline YTH 108 & YP4 & 89 & 92 & 69.1 & 73.9 & 25.8 & 26.2 & 1.3 & 1.4 & 36.4 & 42.6 & 17.8 & 17.7 & 147.4 & 133.4 & 2.6 & 2.5 \\
\hline YTH 112 & YP4 & 88 & 91 & 75.3 & 83.1 & 26.1 & 26.7 & 1.4 & 1.5 & 37.1 & 44.8 & 16.6 & 16.7 & 134.0 & 140.5 & 2.5 & 2.5 \\
\hline YTH 113 & YP4 & 89 & 92 & 75.9 & 84.2 & 26.8 & 26.9 & 1.4 & 1.5 & 38.4 & 44.5 & 17.7 & 15.6 & 164.8 & 156.1 & 2.5 & 2.6 \\
\hline H 114 & YP & 87 & 92 & 74.0 & 82.4 & 26.2 & 27.7 & 1.4 & 1.5 & 39.3 & 46.2 & 17.0 & 16.1 & 173.9 & 175 & 2.5 & 2.5 \\
\hline YTH 118 & YP4 & 87 & 90 & 73.7 & 75. & 26.5 & 26.8 & 1.4 & 1. & 39.3 & 44.5 & 18.8 & 15.3 & 160.6 & 169.0 & 2.5 & 2.5 \\
\hline YTH 119 & YP4 & 87 & 90 & 71.5 & 74.2 & 26.3 & 26.2 & 1.5 & 1.5 & 40.9 & 43.9 & 16.9 & 14.6 & 157.0 & 169.9 & 2.4 & 2.4 \\
\hline YTH 122 & YP4 & 86 & 91 & 74.0 & 78.6 & 26.6 & 27.6 & 1.5 & 1.6 & 39.9 & 47.7 & 16.7 & 14.1 & 175.2 & 169.7 & 2.4 & 2.4 \\
\hline YTH 123 & YP4 & 88 & 92 & 73.4 & 77.5 & 25.8 & 26.6 & 1.3 & 1.4 & 36.1 & 42.6 & 17.7 & 17.2 & 137.8 & 135.9 & 2.8 & 2.7 \\
\hline I 124 & & 90 & 93 & 75.4 & 81.3 & 26.1 & 27.6 & 1.3 & 1.4 & 37.2 & 43.7 & 17.8 & 15.7 & 148.9 & 140 & 2.4 & 2.6 \\
\hline YTH 125 & YP4 & 86 & 91 & 74.0 & 81.1 & 26.7 & 28.4 & 1.3 & 1.4 & 38.3 & 44.2 & 18.2 & 15.9 & 136.6 & 134.9 & 2.6 & 2.6 \\
\hline YP5 & & 93 & 91 & 70.9 & 69.3 & 23.6 & 23.9 & 1.4 & 1.4 & 38.4 & 38.1 & 13.5 & 9.8 & 150.7 & 147.2 & 2.8 & 2.8 \\
\hline YTH 174 & YP5 & 90 & 91 & 55.7 & 60.7 & 21.5 & 22.6 & 1.1 & 1.2 & 23.9 & 28.7 & 19.5 & 19.9 & 96.7 & 95.2 & 2.6 & 2.5 \\
\hline YTH 180 & & 93 & 93 & 59.4 & 59.1 & 23.5 & 24.5 & 1.1 & 1.2 & 25.9 & 31.4 & 23.2 & 23.6 & 103.5 & 100.6 & 2.4 & 2.4 \\
\hline YTH 183 & YP5 & 84 & 89 & 66.4 & 77.2 & 24.3 & 25.4 & 1.2 & 1. & 35.4 & 40.7 & 20.6 & 18.7 & 131.2 & 117.0 & 2.7 & 2.9 \\
\hline YTH 186 & YP5 & 92 & 93 & 78.3 & 86.8 & 24.8 & 25.6 & 1.5 & 1.5 & 34.1 & 38.1 & 19.0 & 18.1 & 131.8 & 120.3 & 2.6 & 2.5 \\
\hline YTH 187 & YP5 & 90 & 92 & 68.5 & 73.8 & 25.3 & 26.4 & 1.4 & 1.4 & 32.1 & 36.9 & 17.9 & 16.7 & 130.8 & 123.1 & 3.0 & 2.5 \\
\hline YTH 191 & YP5 & 87 & 91 & 75.3 & 85.2 & 26.1 & 26.4 & 1.5 & 1.6 & 40.4 & 43.0 & 19.6 & 17.2 & 158.1 & 148.3 & 2.4 & 2.5 \\
\hline YTH 196 & & 89 & 91 & 59.8 & 57.7 & 24.7 & 26.1 & 1.4 & 1. & 30.0 & 34.1 & 21.3 & 22.7 & 120.9 & 133.7 & 2.7 & 2.7 \\
\hline YTH 197 & YP5 & 86 & 90 & 47.8 & 48.5 & 22.2 & 23.8 & 1.1 & 1.1 & 27.6 & 31.3 & 26.0 & 25.4 & 89.5 & 84.7 & 2.6 & 2.7 \\
\hline YTH 198 & YP5 & 87 & 89 & 49.5 & 52.8 & 22.3 & 22.6 & 1.1 & 1.2 & 24.9 & 29.1 & 25.9 & 21.4 & 97.7 & 82.4 & 2.7 & 2.7 \\
\hline YTH 199 & YP5 & 89 & 93 & 67.7 & 76.2 & 26.3 & 26.5 & 1.4 & 1.4 & 39.5 & 42.8 & 19.7 & 17.7 & 155.6 & 120.8 & 2.8 & 2.8 \\
\hline YTH 200 & YP5 & 82 & 88 & 55.5 & 65.8 & 23.3 & 23.9 & 1.0 & 1.1 & 31.0 & 34.6 & 20.0 & 18.2 & 122.2 & 106.9 & 2.8 & 2.7 \\
\hline YTH 203 & YP5 & 83 & 88 & 57.2 & 63.4 & 23.3 & 25.0 & 1.1 & 1. & 29.6 & 35.6 & 21.9 & 19.8 & 119.6 & 114.1 & 2.8 & 2.6 \\
\hline YTH 205 & YP5 & 91 & 94 & 55.7 & 55.7 & 24.5 & 26.0 & 1.0 & 1.1 & 28.3 & 31.0 & 20.9 & 22.0 & 122.2 & 125.2 & 2.6 & 2.3 \\
\hline YTH 210 & YP5 & 85 & 87 & 53.3 & 58.9 & 22.8 & 23.2 & 1.1 & 1.2 & 29.9 & 32.8 & 27.8 & 22.1 & 98.2 & 90.2 & 2.2 & 2.2 \\
\hline YTH 213 & YP5 & 83 & 85 & 60.9 & 69.7 & 24.2 & 25.3 & 1.3 & 1.6 & 32.9 & 38.4 & 19.2 & 15.4 & 107.6 & 114.5 & 2.6 & 2.6 \\
\hline YTH 218 & YP5 & 89 & 90 & 58.1 & 63.3 & 23.7 & 25.1 & 1.1 & 1. & 24.3 & 28.4 & 23.3 & 21.8 & 122.2 & 116.3 & 2.4 & 2.3 \\
\hline YTH 222 & YP5 & 92 & 93 & 53.5 & 57.6 & 24.1 & 25.3 & 1.3 & 1. & 34.4 & 39.8 & 22.3 & 17.7 & 149.9 & 141.1 & 2.4 & 2.4 \\
\hline YTH 225 & YP5 & 85 & 88 & 58.9 & 62.2 & 22.9 & 24.2 & 1.2 & 1.2 & 28.1 & 33.0 & 23.7 & 19.6 & 101.2 & 96.2 & 2.7 & 2.7 \\
\hline YTH 226 & YP5 & 82 & 85 & 56.0 & 61.9 & 22.0 & 23.6 & 1.1 & 1.3 & 28.3 & 32.6 & 21.0 & 17.1 & 86.4 & 88.8 & 2.6 & 2.7 \\
\hline
\end{tabular}


D. Fujita et al.

Table 3. Continued.

\begin{tabular}{|c|c|c|c|c|c|c|c|c|c|c|c|c|c|c|c|c|c|}
\hline \multirow{3}{*}{$\begin{array}{l}2005 W S \\
\text { Entry No. }\end{array}$} & \multirow{3}{*}{$\begin{array}{l}\text { Donor } \\
\text { variety }{ }^{\mathrm{a}}\end{array}$} & \multicolumn{16}{|c|}{ Average of agronomic traits in each INL } \\
\hline & & \multicolumn{2}{|c|}{ DTH(day) } & \multicolumn{2}{|c|}{$\mathrm{CL}(\mathrm{cm})$} & \multicolumn{2}{|c|}{ PL $(\mathrm{cm})$} & \multicolumn{2}{|c|}{$\mathrm{LW}(\mathrm{cm})$} & LL( & $\mathrm{cm})$ & $\mathrm{P}$ & & & & & \\
\hline & & DS & WS & DS & WS & DS & WS & DS & WS & DS & WS & DS & WS & DS & WS & DS & WS \\
\hline YP6 & & 99 & 98 & 80.8 & 79.1 & 28.6 & 29.4 & 2.1 & 2.1 & 43.8 & 44.1 & 11.2 & 8.3 & 172.0 & 152.3 & 3.0 & 3.0 \\
\hline YTH 243 & YP6 & 88 & 92 & 64.4 & 73.7 & 23.5 & 24.7 & 1.3 & 1.3 & 33.8 & 35.9 & 19.2 & 16.9 & 115.4 & 103.1 & 2.6 & 2.6 \\
\hline YTH 245 & YP6 & 93 & 98 & 72.3 & 79.6 & 24.4 & 25.8 & 1.3 & 1.3 & 31.8 & 35.5 & 18.9 & 16.7 & 120.5 & 113.8 & 2.7 & 2.6 \\
\hline YTH 246 & YP6 & 94 & 99 & 72.0 & 80.8 & 24.8 & 25.6 & 1.3 & 1.3 & 33.0 & 36.9 & 20.7 & 16.9 & 125.5 & 110.1 & 2.7 & 2.6 \\
\hline YTH 247 & YP6 & 95 & 99 & 71.3 & 77.9 & 23.7 & 24.5 & 1.4 & 1.3 & 29.8 & 35.7 & 20.0 & 17.0 & 118.4 & 110.2 & 2.6 & 2.5 \\
\hline YTH 252 & YP6 & 92 & 96 & 59.7 & 67.2 & 23.6 & 24.6 & 1.3 & 1.4 & 29.4 & 33.2 & 19.6 & 17.4 & 95.8 & 99.2 & 2.6 & 2.6 \\
\hline YTE 254 & YP6 & 86 & 89 & 64.8 & 70.8 & 24.0 & 24.2 & 1.3 & 1.4 & 34.4 & 36.0 & 22.1 & 18.8 & 108.6 & 92.8 & 2.7 & 2.6 \\
\hline YTH 255 & YP6 & 89 & 94 & 64.4 & 68.2 & 24.7 & 26.7 & 1.4 & 1.3 & 31.3 & 34.7 & 19.6 & 16.8 & 119.8 & 107.0 & 2.6 & 2.5 \\
\hline YP7 & & 80 & 78 & 64.4 & 58.9 & 20.1 & 20.0 & 1.0 & 0.9 & 33.6 & 33.6 & 19.3 & 12.0 & 123.6 & 103.7 & 3.2 & 3.2 \\
\hline YTH 259 & YP7 & 75 & 79 & 54.5 & 60.5 & 18.9 & 20.7 & 1.1 & 1.1 & 30.3 & 36.6 & 30.0 & 25.1 & 73.7 & 104.4 & 2.7 & 2.8 \\
\hline YTH 260 & YP7 & 78 & 80 & 54.0 & 60.4 & 18.9 & 19.4 & 1.1 & 1.1 & 30.6 & 34.8 & 28.4 & 27.1 & 67.9 & 65.5 & 2.7 & 2.7 \\
\hline YTH 262 & YP7 & 76 & 79 & 52.1 & 58.6 & 20.0 & 20.7 & 1.2 & 1.2 & 30.4 & 38.7 & 28.9 & 22.0 & 67.0 & 60.2 & 2.7 & 2.7 \\
\hline YTH 264 & YP7 & 76 & 80 & 51.1 & 59.8 & 20.8 & 21.0 & 1.2 & 1.2 & 29.6 & 36.6 & 27.7 & 23.0 & 69.1 & 66.1 & 2.7 & 2.8 \\
\hline YTH 266 & YP7 & 79 & 80 & 61.1 & 66.1 & 21.7 & 21.8 & 1.1 & 1.2 & 32.7 & 36.5 & 24.2 & 19.2 & 106.1 & 93.7 & 2.5 & 2.6 \\
\hline YTH 269 & YP7 & 83 & 84 & 61.8 & 64.5 & 20.8 & 21.5 & 1.3 & 1.3 & 31.7 & 35.8 & 27.0 & 21.6 & 98.3 & 88.1 & 2.4 & 2.4 \\
\hline YTH 270 & YP7 & 84 & 86 & 67.6 & 67.3 & 23.3 & 23.5 & 1.2 & 1.2 & 34.7 & 38.0 & 20.0 & 16.9 & 154.6 & 126.8 & 2.3 & 2.3 \\
\hline YTH 271 & YP7 & 85 & 87 & 69.8 & 70.9 & 23.6 & 24.1 & 1.3 & 1.3 & 34.4 & 36.5 & 20.9 & 16.4 & 148.5 & 127.2 & 2.4 & 2.5 \\
\hline YTH 272 & YP7 & 80 & 82 & 55.4 & 61.8 & 20.0 & 19.4 & 1.2 & 1.2 & 31.4 & 32.5 & 28.6 & 25.9 & 74.5 & 70.3 & 2.5 & 2.5 \\
\hline YTH 277 & YP7 & 76 & 82 & 53.9 & 59.0 & 19.3 & 20.0 & 1.1 & 1.1 & 29.3 & 35.3 & 25.4 & 21.0 & 83.0 & 78.3 & 2.6 & 2.5 \\
\hline YTH 279 & YP7 & 78 & 81 & 56.6 & 62.3 & 20.5 & 20.5 & 1.1 & 1.1 & 32.9 & 35.1 & 24.7 & 21.3 & 103.1 & 88.2 & 2.5 & 2.5 \\
\hline YP8 & & 102 & 98 & 70.2 & 70.2 & 22.0 & 20.6 & 1.6 & 1.5 & 39.1 & 39.9 & 11.7 & 11.9 & 190.1 & 149.7 & 2.8 & 2.6 \\
\hline YTH 284 & YP8 & 86 & 89 & 65.9 & 66.2 & 24.0 & 24.1 & 1.4 & 1.4 & 38.3 & 39.6 & 23.2 & 19.6 & 150.5 & 121.5 & 2.3 & 2.3 \\
\hline YTH 287 & YP8 & 89 & 91 & 72.9 & 76.7 & 24.0 & 23.8 & 1.4 & 1.4 & 38.4 & 42.8 & 19.3 & 17.1 & 175.3 & 161.5 & 2.5 & 2.5 \\
\hline YTH 288 & YP8 & 93 & 94 & 77.0 & 83.5 & 25.8 & 26.4 & 1.4 & 1.4 & 34.9 & 38.5 & 18.9 & 15.8 & 189.5 & 176.7 & 2.5 & 2.5 \\
\hline YTH 289 & YP8 & 87 & 88 & 60.8 & 64.4 & 24.2 & 23.4 & 1.2 & 1.2 & 34.3 & 35.4 & 24.2 & 21.0 & 126.1 & 97.5 & 2.5 & 2.4 \\
\hline YTH 292 & YP8 & 84 & 85 & 63.0 & 70.7 & 24.3 & 24.7 & 1.1 & 1.2 & 33.5 & 37.5 & 20.3 & 16.8 & 124.3 & 124.4 & 2.5 & 2.5 \\
\hline YTH 297 & YP8 & 81 & 86 & 60.8 & 65.9 & 22.9 & 24.7 & 1.2 & 1.3 & 33.6 & 39.0 & 20.3 & 16.1 & 138.9 & 130.6 & 2.4 & 2.4 \\
\hline YTH 302 & YP8 & 85 & 87 & 66.1 & 71.3 & 22.8 & 23.7 & 1.2 & 1.3 & 35.2 & 39.6 & 22.6 & 19.4 & 123.2 & 119.6 & 2.5 & 2.5 \\
\hline YTH 303 & YP8 & 86 & 88 & 69.3 & 71.2 & 22.6 & 22.7 & 13 & 1.3 & 36.5 & 40.1 & 19.9 & 16.4 & 143.9 & 136.5 & 2.5 & 2.5 \\
\hline YTH 304 & YP8 & 82 & 89 & 65.9 & 70.9 & 24.6 & 25.1 & 1.4 & 1.4 & 39.2 & 41.2 & 18.3 & 13.7 & 167.3 & 151.8 & 2.4 & 2.4 \\
\hline YTH 306 & YP8 & 79 & 83 & 65.2 & 70.8 & 24.5 & 24.6 & 1.2 & 1.3 & 35.8 & 39.4 & 20.8 & 16.3 & 136.0 & 137.6 & 2.6 & 2.5 \\
\hline YP9 & & 98 & 89 & 69.2 & 67.9 & 23.9 & 22.3 & 1.6 & 1.5 & 38.6 & 36.7 & 10.1 & 8.7 & 167.6 & 132.5 & 2.7 & 2.9 \\
\hline YTH 318 & YP9 & 90 & 91 & 73.6 & 74.0 & 23.1 & 24.4 & 1.6 & 1.6 & 38.1 & 44.3 & 18.7 & 17.3 & 154.5 & 136.0 & 2.4 & 2.4 \\
\hline YTH 319 & YP9 & 87 & 89 & 64.4 & 66.3 & 23.7 & 23.5 & 1.3 & 1.3 & 35.8 & 39.1 & 19.6 & 19.0 & 118.1 & 105.6 & 2.6 & 2.7 \\
\hline YTH 323 & YP9 & 92 & 88 & 75.6 & 79.1 & 23.5 & 23.2 & 1.7 & 1.7 & 36.1 & 41.5 & 16.1 & 16.7 & 144.5 & 135.2 & 2.4 & 2.4 \\
\hline YTH 324 & YP9 & 84 & 86 & 65.9 & 74.6 & 23.2 & 23.7 & 1.2 & 1.4 & 32.2 & 37.1 & 20.5 & 18.6 & 108.3 & 98.4 & 2.6 & 2.6 \\
\hline YTH 325 & YP9 & 87 & 88 & 71.6 & 71.0 & 23.1 & 23.2 & 1.5 & 1.6 & 37.4 & 42.0 & 17.1 & 15.8 & 162.4 & 145.4 & 2.4 & 2.5 \\
\hline YTH 326 & YP9 & 86 & 86 & 70.1 & 72.5 & 23.1 & 23.4 & 1.4 & 1.5 & 38.0 & 41.8 & 20.6 & 17.3 & 141.8 & 140.2 & 2.5 & 2.5 \\
\hline YTH 328 & YP9 & 85 & 87 & 66.7 & 70.2 & 22.9 & 23.4 & 1.2 & 1.3 & 34.5 & 38.9 & 19.7 & 18.6 & 134.5 & 115.9 & 2.6 & 2.7 \\
\hline YP10 & & 66 & - & 54.8 & 53.3 & 17.8 & 17.8 & 0.9 & 0.9 & 24.0 & 28.2 & 23.1 & 17.8 & 47.3 & 55.1 & 2.6 & 2.6 \\
\hline YTH 332 & YP10 & 85 & 87 & 67.7 & 76.3 & 25.0 & 25.3 & 1.3 & 1.3 & 34.7 & 38.2 & 21.0 & 18.8 & 125.3 & 110.0 & 2.6 & 2.6 \\
\hline YTH 336 & YP10 & 79 & 85 & 52.5 & 61.6 & 21.6 & 22.7 & 1.1 & 1.2 & 27.6 & 32.7 & 21.6 & 20.8 & 101.6 & 109.1 & 2.9 & 2.6 \\
\hline YTH 339 & YP10 & 81 & 87 & 52.7 & 65.3 & 21.1 & 21.4 & 1.1 & 1.3 & 28.4 & 31.9 & 24.0 & 20.5 & 78.9 & 80.7 & 2.8 & 2.9 \\
\hline YTH 342 & YP10 & 81 & 87 & 57.7 & 68.7 & 22.9 & 23.9 & 1.1 & 1.3 & 31.9 & 38.0 & 19.9 & 18.7 & 112.5 & 94.6 & 3.2 & 3.2 \\
\hline YTH 344 & YP10 & 85 & 88 & 68.0 & 74.5 & 25.0 & 27.0 & 1.2 & 1.3 & 34.8 & 40.9 & 18.6 & 17.2 & 132.8 & 126.3 & 2.7 & 2.6 \\
\hline YTH 351 & YP10 & 81 & 86 & 57.4 & 67.6 & 22.7 & 24.8 & 1.1 & 1.3 & 32.8 & 38.0 & 19.3 & 17.9 & 89.6 & 95.3 & 2.8 & 3.0 \\
\hline YTH 353 & YP10 & 82 & 84 & 55.6 & 60.7 & 20.5 & 21.7 & 1.2 & 1.3 & 31.0 & 36.1 & 21.4 & 19.3 & 79.9 & 84.9 & 2.8 & 2.8 \\
\hline YTH 360 & YP10 & 81 & 86 & 62.8 & 72.1 & 22.9 & 23.3 & 1.2 & 1.3 & 33.8 & 36.0 & 19.8 & 18.6 & 110.0 & 103.6 & 3.0 & 2.6 \\
\hline YTH 362 & YP10 & 82 & 88 & 52.1 & 61.3 & 20.8 & 21.5 & 1.1 & 1.2 & 28.5 & 32.4 & 23.7 & 20.6 & 83.9 & 81.6 & 2.4 & 2.5 \\
\hline YTH 363 & YP10 & 84 & 90 & 48.2 & 56.0 & 22.0 & 23.4 & 1.2 & 1.3 & 30.6 & 36.3 & 19.6 & 17.1 & 90.7 & 89.0 & 2.8 & 2.8 \\
\hline YTH 368 & YP10 & 82 & 87 & 50.3 & 63.1 & 20.8 & 22.1 & 1.1 & 1.3 & 29.5 & 34.4 & 18.0 & 16.0 & 75.1 & 89.8 & 3.0 & 2.9 \\
\hline YP11 & & 105 & 99 & 58.7 & 60.8 & 23.6 & 24.5 & 1.8 & 1.9 & 37.7 & 39.3 & 12.2 & 7.6 & 180.0 & 186.3 & 2.6 & 2.4 \\
\hline YTH 133 & YP11 & 88 & 90 & 63.6 & 62.3 & 23.8 & 23.3 & 1.2 & 1. & 34.3 & 35.9 & 21.4 & 18.3 & 106.2 & 97.6 & 2.6 & 2.6 \\
\hline YTH 135 & YP11 & 86 & 89 & 68.0 & 64.9 & 26.5 & 26.5 & 1.5 & 1.6 & 37.5 & 38.9 & 20.0 & 18.3 & 143.4 & 131.9 & 2.6 & 2.7 \\
\hline YTH 137 & YP11 & 88 & 90 & 66.1 & 69.9 & 25.3 & 25.2 & 1.4 & 1.5 & 40.4 & 42.0 & 19.5 & 19.3 & 142.5 & 133.4 & 2.5 & 2.4 \\
\hline YTH 146 & YP11 & 84 & 88 & 59.5 & 64.7 & 23.2 & 23.1 & 1.3 & 1.3 & 33.6 & 34.7 & 23.2 & 20.7 & 114.7 & 88.0 & 2.7 & 2.7 \\
\hline YTH 150 & YP11 & 90 & 90 & 69.7 & 72.6 & 26.4 & 26.3 & 1.5 & 1.6 & 40.6 & 44.7 & 20.5 & 17.0 & 155.2 & 129.1 & 2.7 & 2.7 \\
\hline YTH 153 & YP11 & 87 & 88 & 66.6 & 73.9 & 24.4 & 25.2 & 1.3 & 1.5 & 34.9 & 40.5 & 21.5 & 17.3 & 117.4 & 107.3 & 2.6 & 2.7 \\
\hline YTH 155 & YP11 & 88 & 90 & 69.7 & 73.2 & 26.1 & 26.6 & 1.5 & 1.6 & 40.2 & 43.0 & 20.6 & 13.7 & 167.7 & 136.0 & 2.5 & 2.5 \\
\hline YTH 157 & YP11 & 87 & 90 & 65.5 & 70.4 & 25.2 & 25.4 & 1.5 & 1.6 & 37.7 & 40.3 & 21.3 & 16.5 & 147.0 & 136.5 & 2.6 & 2.6 \\
\hline YTH 160 & YP11 & 87 & 90 & 68.5 & 74.1 & 25.6 & 25.8 & 1.6 & 1.7 & 38.6 & 42.1 & 19.1 & 15.9 & 161.1 & 130.9 & 2.6 & 2.6 \\
\hline YTH 162 & YP11 & 88 & 90 & 69.7 & 73.9 & 25.4 & 26.2 & 1.5 & 1.6 & 36.9 & 42.7 & 19.6 & 17.4 & 148.6 & 132.() & 2.5 & 2.4 \\
\hline YTH 165 & YP11 & 88 & 90 & 67.7 & 70.4 & 26.0 & 25.4 & 1.5 & 1.6 & 38.4 & 41.6 & 20.2 & 17.4 & 158.2 & 140.4 & 2.6 & 2.5 \\
\hline
\end{tabular}

DTH: days to heading, CL: culm length, PL: panicle length, LW: leaf width, LL: leaf length, PN: panicle number per plant, GW:

100-grain weight, TSN: total spikelet number per panicle.

The phenotypic averages of DS in 2006, 2007 and 2008 and of WS in 2005, 2006 and 2007 were shown.

a) See Table 1 . 


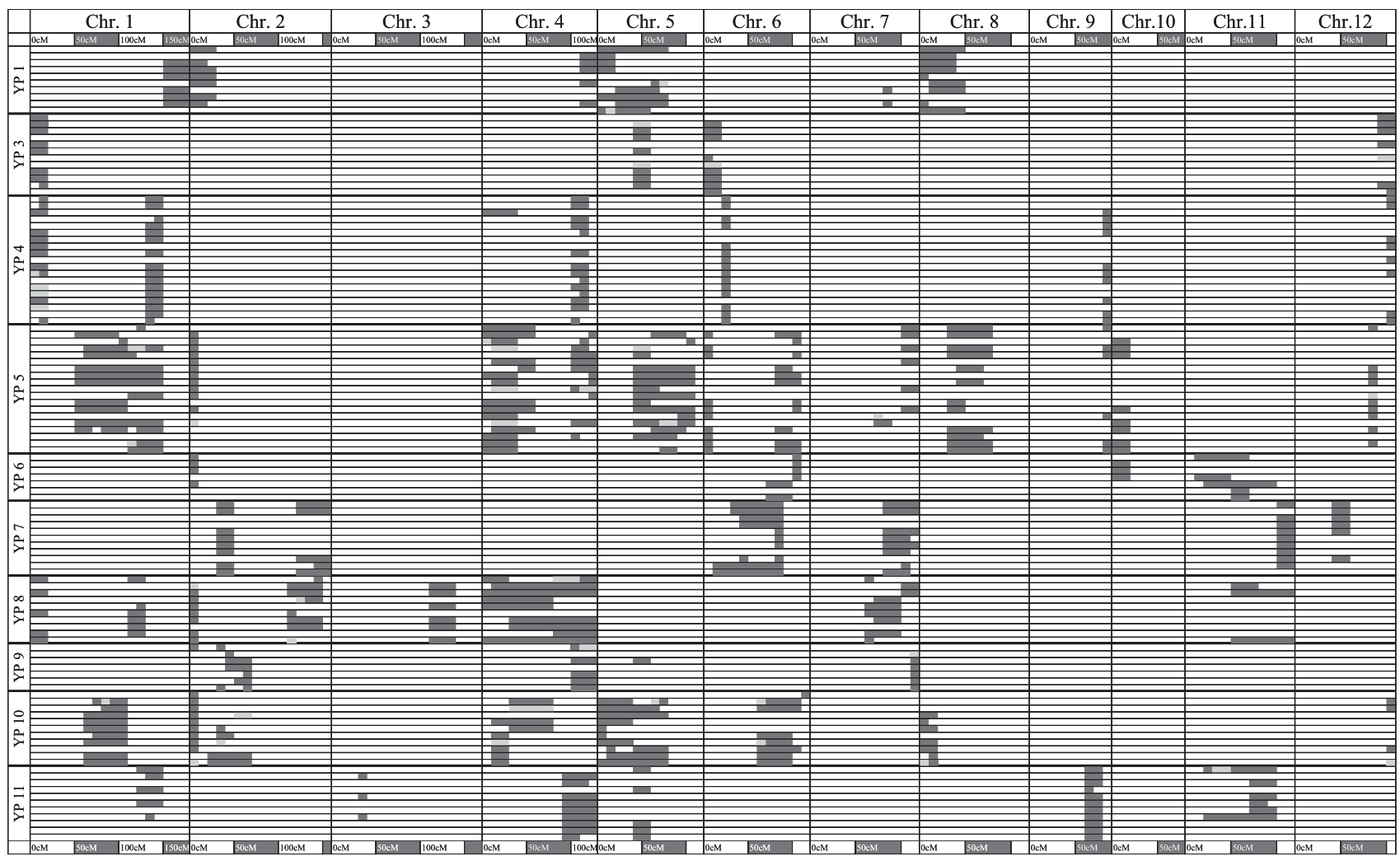

Fig. 2. Graphical genotypes of 117 introgression lines derived from crosses between IR64 and 10 donor parents The black regions denote the homozygous for donor allele; the white regions denote the homozygous for IR 64 allele; the gray regions denote the heterozygous alleles.

GW of three INLs in DS was heavier than that of IR64: by $0.2 \mathrm{~g}$ in YTH199 (YP5), by $0.4 \mathrm{~g}$ in YTH33 (YP1), and by $0.5 \mathrm{~g}$ in YTH368 (YP10) (Fig. 4B). YTH33 had an associated region YP1-GW5 for heavy GW. TSN of three INLs were lower than that of IR64, while that of seven INLs was higher (Fig. 4C). TSN of three INLs in DS were lower than that of IR64: by 39 in YTH13 (YP 1), by 40 in YTH264 (YP7) and by 30 in YTH339 (YP 10). TSN of seven INLs in DS was higher than that of IR64: by 58 in YTH63 (YP3), by 91 in YTH83 (YP4), by 49 in YTH191 (YP5), by 46 in YTH270 (YP7), by 81 in YTH288 (YP8), by 54 in YTH325 (YP9), and by 52 in YTH160 (YP11). Three INLs which showed lower TSN compared with IR64 had the associated regions: YTH264 with YP7-TSN6, YTH13 with YP1-TSN1 and YP1-TSN5, and YTH339 with YP10-TSN6. On the other hand, five INLs which showed higher TSN compared with IR64 had the associated regions: YTH191 with YP5-TSN4, YTH160 with YP11-TSN4, YTH325 with YP9-TSN4, YTH288 with YP8-TSN4, and YTH83 with YP4-TSN4. The INLs which showed unique agronomic traits: short and long DTH, high and low TSN, and heavy GW, were found across multiple sib groups.

\section{Discussion}

In our previous study, association analyses between agronomic traits and introgressed segments in the 334 INLs were conducted using SSR markers ${ }^{2}$. As a result, two regions for short DTH in YP1 and YP7 and five regions for long DTH in YP1, YP3, YP5, YP8, and YP10 were identified. The results estimated that several regions from the 334 INLs coincided with QTLs and genes which have been previously reported. For example, YTH13 which showed short DTH had an introgressed segment at the same region of the Hd5 locus on chromosome $8^{13}$. YTH59 which showed long DTH had introgressed segments at the similar location of $\mathrm{Hd} 3 \mathrm{a}$ and $H d 3 b$ on chromosome $6^{8,17}$. Thus, these associated regions in these INLs which were responsible for DTH were thought to correspond with results of previous studies. A region for GW in YP1 contributed to increase $\mathrm{GW}$ in previous study and was located on the centromere region of chromosome 5. From this region of chromosome 5, qSW5 for grain size related to $\mathrm{GW}$ was isolated $^{23}$. This fact suggested that YTH33 (derived from YP1) for heavy GW might have qSW5. Although an associated region for GW in analysis of variation was not 


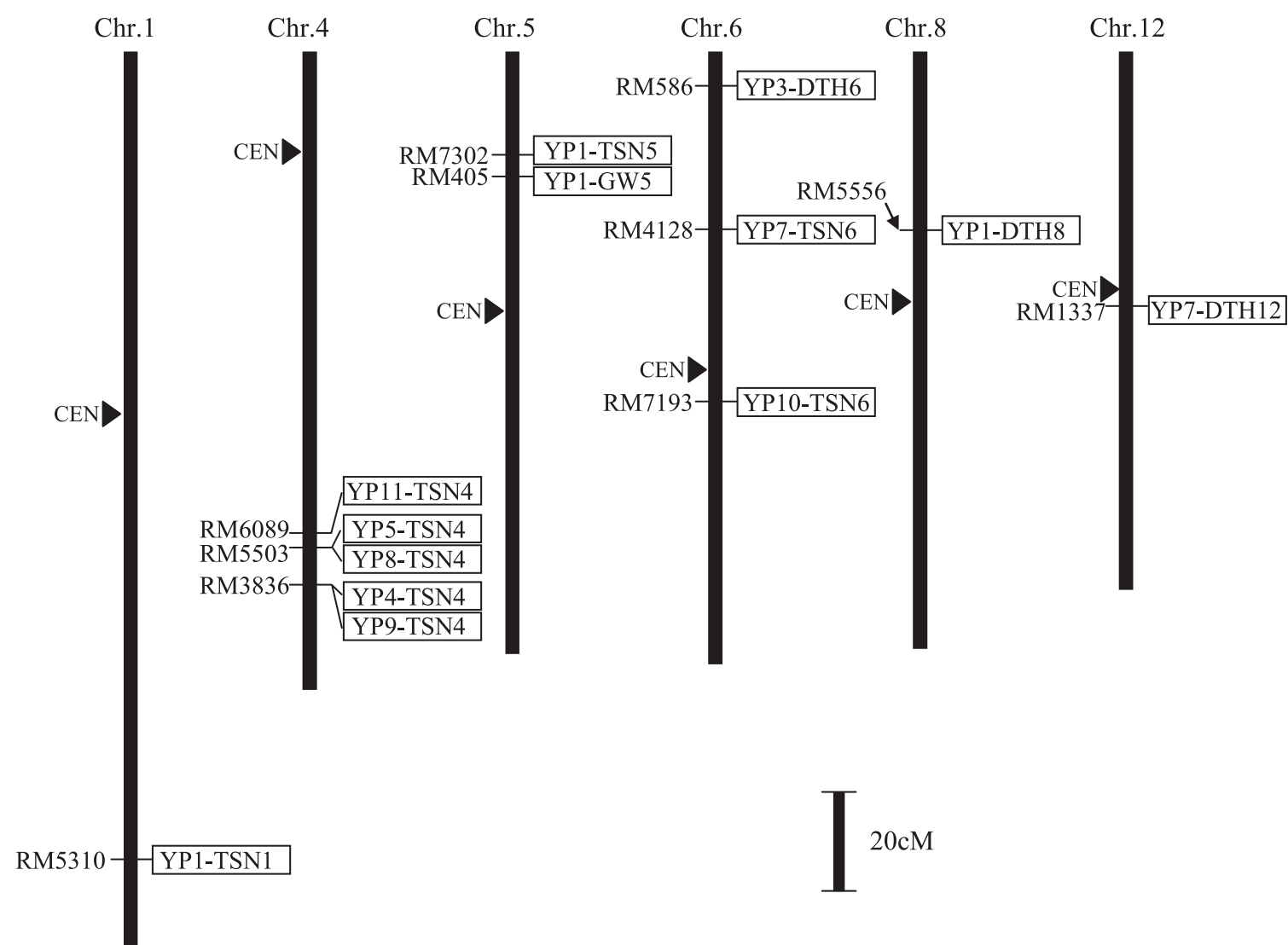

Fig. 3. Chromosomal locations of associated regions for agronomic traits in $\mathbf{3 3 4}$ introgression lines

The SSR markers associated with each agronomic trait in analysis of variation were quoted from Fujita et al. (2009) $)^{2}$. The rectangles indicate the regions of introgressed segments associated with agronomic traits. The name for an associated region in rectangles, for example, YP1-DTH8, represented the donor parent YP1, followed by the trait DTH, and chromosome number 8.

identified in YTH199 from YP5 and YTH368 from YP 10 which showed heavy GW, these lines had introgressed segments around $q S W 5$ on chromosome 5. This result indicated that YTH199 and YTH368 may have $q S W 5$. Additionally, a region contributing to increase TSN was detected in association analysis. The region for high TSN on the long arm of chromosome 4 was detected across five sib groups from YP4, YP5, YP8, YP 9, and YP11. In previous study, several QTLs for TSN have been detected on the same region of chromosome

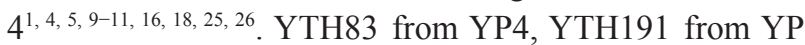
5, YTH288 from YP8, YTH325 from YP9, and YTH160 from YP11 had the common region on the long arm of chromosome 4. Although the associated regions for TSN in most of these INLs have been identified, the associated regions of YTH270 and YTH63 for high TSN have not been detected by analysis of variation. QTL analysis for agronomic traits using segregated populations derived from YTH246, YTH199, YTH368, YTH270, and YTH63, will reveal the location and effect of QTLs and will provide useful genetic information for MAS.
In this study, developed 334 INLs showed unique agronomic traits such as short and long DTH, heavy GW, and low and high TSN. Although the developed INLs are useful as breeding materials and for studying agronomic traits, each INL contained multiple introgressed segments. For instance, near isogenic lines (NILs) for agronomic traits can be easily developed based on these developed INLs. The NILs are more suitable materials for precise genetic studies, including the evaluation of gene effects, selection of enhanced molecular markers which are tightly linked with the target gene, gene expression, and gene isolation. If the target trait is decided, it is easy to develop NILs with IR64 genetic background by MAS using the developed INLs based on the information of associated regions. The NILs for agronomic traits can be used to reveal the gene-environment interaction. Understandably, the developed INLs in a uniform background of IR64 are useful not only as research materials but also as pre-breeding lines. 
A

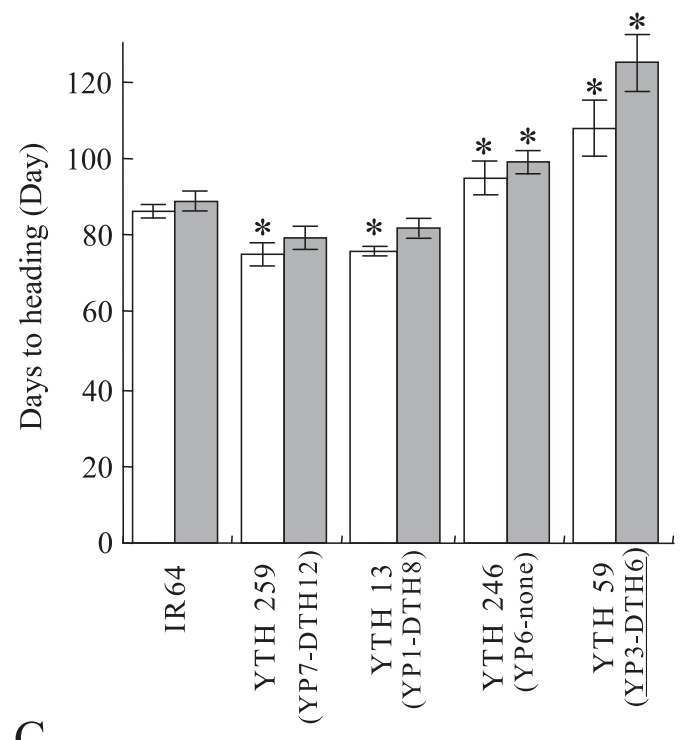

$\mathrm{B}$

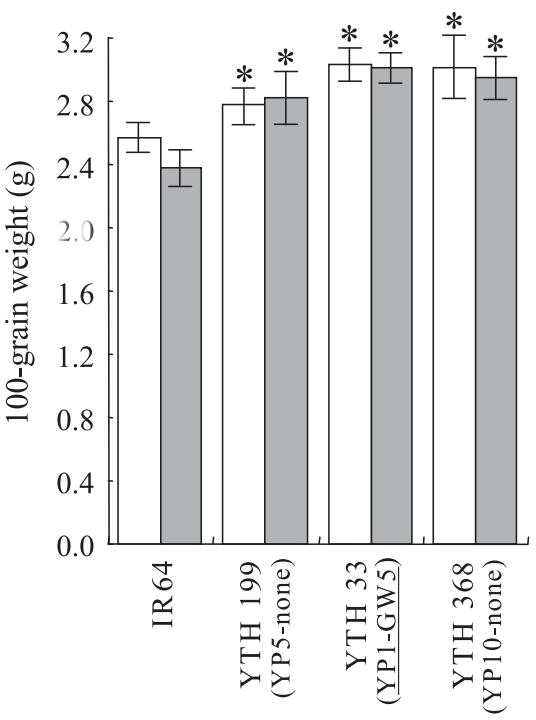

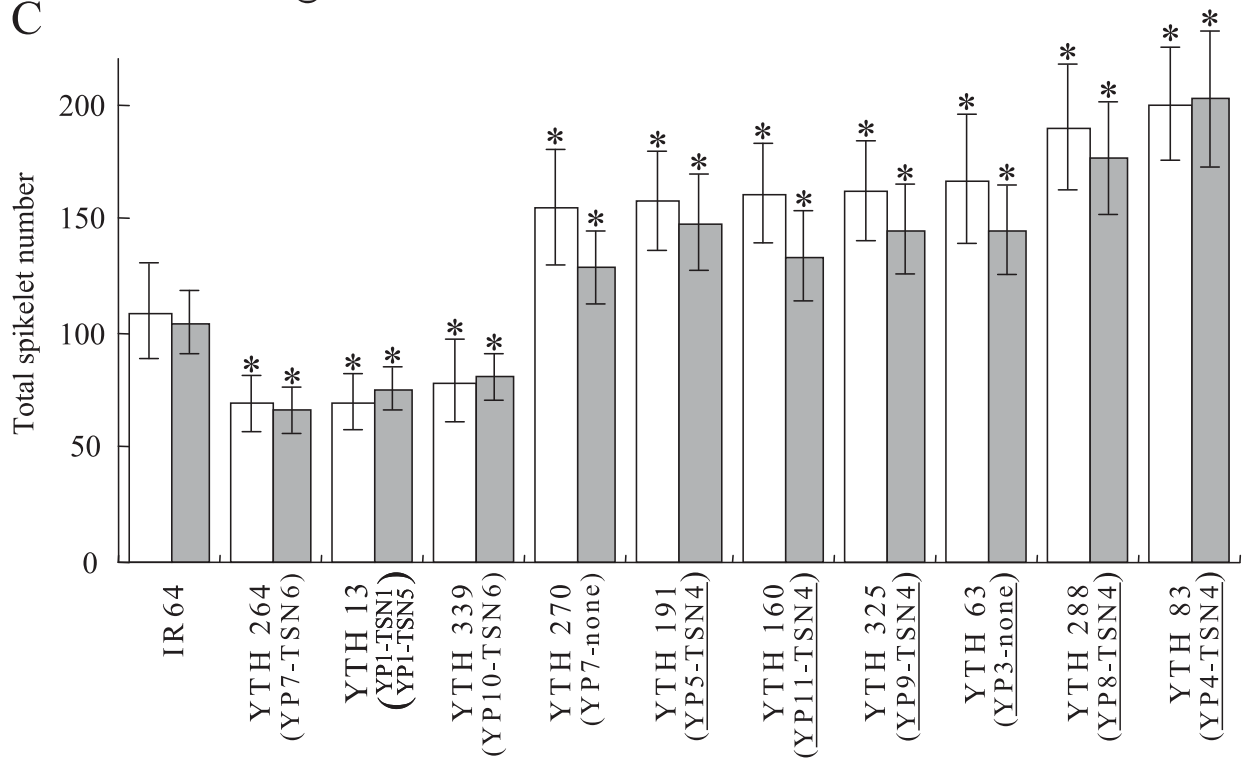

Fig. 4. Comparison for agronomic traits for wet season (WS) in 2007 and dry season (DS) in 2008:

(A) days to heading, (B) 100-grain weight, (C) total spikelet number among typical line

The names in parenthesis indicate the regions associated with agronomic traits at $0.1 \%$ level and were designated as donor varieties followed by the traits and chromosome number. Bars indicate the average of each trait with standard deviation. Asterisks indicate a significant difference between IR64 and the INLs at 5\% level by Dunnett's test.

: DS, : WS.

\section{Acknowledgments}

This paper reports the results obtained in the IRRIJapan Collaborative Research Project phases III, IV and $\mathrm{V}$ supported by the Ministry of Foreign Affairs and the Ministry of Agriculture, Forestry and Fisheries of Japan. We thank Dr. David Mackill and many other staff in IRRI for supporting our breeding program.

\section{References}

1. Brondani, C. et al. (2002) QTL mapping and introgression of yield-related traits from Oryza glumaepatula to cultivated rice (Oryza sativa) using microsatellite markers. Theor. Appl. Genet., 104, 1192-1203.

2. Fujita, D. et al. (2009) Development of introgression lines of an Indica-type rice variety, IR64, for unique agronomic traits and detection of the responsible chromosomal regions. Field Crops Res., 114, 244-254. 
3. Guiderdoni, E. et al. (1992) Anther culture of tropical japonica $\mathrm{x}$ indica hybrids of rice (Oryza sativa L.). Euphytica, 62, 219-224.

4. He, P. et al. (2001) Comparison of molecular linkage maps and agronomic trait loci between DH and RIL populations derived from the same rice cross. Crop Sci., 41, 1240-1246.

5. Hittalmani, S. et al. (2003) Identification of QTL for growth- and grain yield-related traits in rice across nine locations of Asia. Theor. Appl. Genet., 107, 679-690.

6. Huang, N. et al. (1997) RFLP mapping of isozymes, RAPD and QTLs for grain shape, brown planthopper resistance in a doubled haploid rice population. Mol. Breed., 3, 105-113.

7. Khush, G. S. (1987) Rice breeding: past, present and future. J. Genetics, 66, 195-216.

8. Kojima, S. et al. (2002) $H d 3 a$, a rice ortholog of the Arabidopsis FT gene, promotes transition to flowering downstream of $H d l$ under short-day conditions. Plant Cell Physiol., 43, 1096-1105.

9. Lafitte, H. R. et al. (2002) Genetic improvement of rice in aerobic systems: progress from yield to genes. Field Crop Res., 75, 171-190.

10. Lanceras, J. C. et al. (2004) Quantitative trait loci associated with drought tolerance at reproductive stage in rice. Plant Physiology, 135, 384-399.

11. Li, C. et al. (2006) Genetic analysis of rice domestication syndrome with the wild annual species, Oryza nivara. New Phytologist, 170, 185-194.

12. Liao, C. Y. et al. (2001) Effects of genetic background and environment on QTLs and epistasis for rice (Oryza sativa L.) panicle number. Theor. Appl. Genet., 103, 104-111.

13. Lin, H. et al. (2003) Fine mapping and characterization of quantitative traits loci $H d 4$ and $H d 5$ controlling heading date in rice. Breed. Sci., 53, 51-59.

14. Maeda, H. et al. (2003) A new rice cultivar for whole crop silage, "Hoshiaoba". Kinki Chugoku Shikoku Nougyo Kenkyu Center Kenkyu-hokoku (Bull. WeNARC), 2, 83-98 [In Japanese with English summary].
15. McCouch, S. R. et al. (2002) Development and mapping of 2240 new SSR markers for rice (Oryza sativa L.). DNA Res., 9, 199-207.

16. Mei, H. W. et al. (2005) Gene actions of QTLs affecting several agronomic traits resolved in a recombinant inbred rice population and two backcross populations. Theor. Appl. Genet., 110, 649-659.

17. Monna, L. et al. (2002) Genetic dissection of a genomic region for a quantitative trait locus, $\mathrm{Hd} 3$, into two loci, $H d 3 a$ and $H d 3 b$, controlling heading date in rice. Theor. Appl. Genet., 104, 772-778.

18. Onishi, K. et al. (2007) A QTL cluster for plant architecture and its ecological significance in Asian wild rice. Breed. Sci., 57, 7-16.

19. Peng, S. et al. (1999) Yield potential trends of tropical rice since the release of IR8 and the challenge of increasing rice yield potential. Crop Sci., 39, 1552-1559.

20. Peng, S. \& Khush, G. S. (2003) Four decades of breeding for varietal improvement of irrigated lowland rice in the International Rice Research Institute. Plant Prod. Sci., 6, 157-164.

21. Rogers, S. O. \& Bendich, A. J. (1988) Extraction of DNA from plant tissues. Plant Mol. Biol. Manual, A6, $1-10$.

22. Sallaud, C. et al. (2003) Identification of five new blast resistance genes in the highly blast-resistant rice variety IR64 using a QTL mapping strategy. Theor. Appl. Genet., 106, 794-803.

23. Shomura, A. et al. (2008) Deletion in a gene associated with grain size increased yields during rice domestication. Nature Genet., 40, 1023-1028.

24. Wu, J. L. et al. (2005) Chemical- and irradiationinduced mutants of indica rice IR64 for forward and reverse genetics. Plant Mol. Biol., 59, 85-97.

25. Zhuang, J. Y. et al. (1997) Analysis of QTL3 environment interaction for yield components and plant height in rice. Theor. Appl. Genet., 95, 799-808.

26. Zou, G. H. et al. (2005) Grain yield responses to moisture regimes in a rice population: association among traits and genetic markers. Theor. Appl. Genet., 112, 106-113. 